\title{
Current Biology \\ Oil Body Formation in Marchantia polymorpha Is Controlled by MpC1HDZ and Serves as a Defense against Arthropod Herbivores
}

\section{Graphical Abstract}

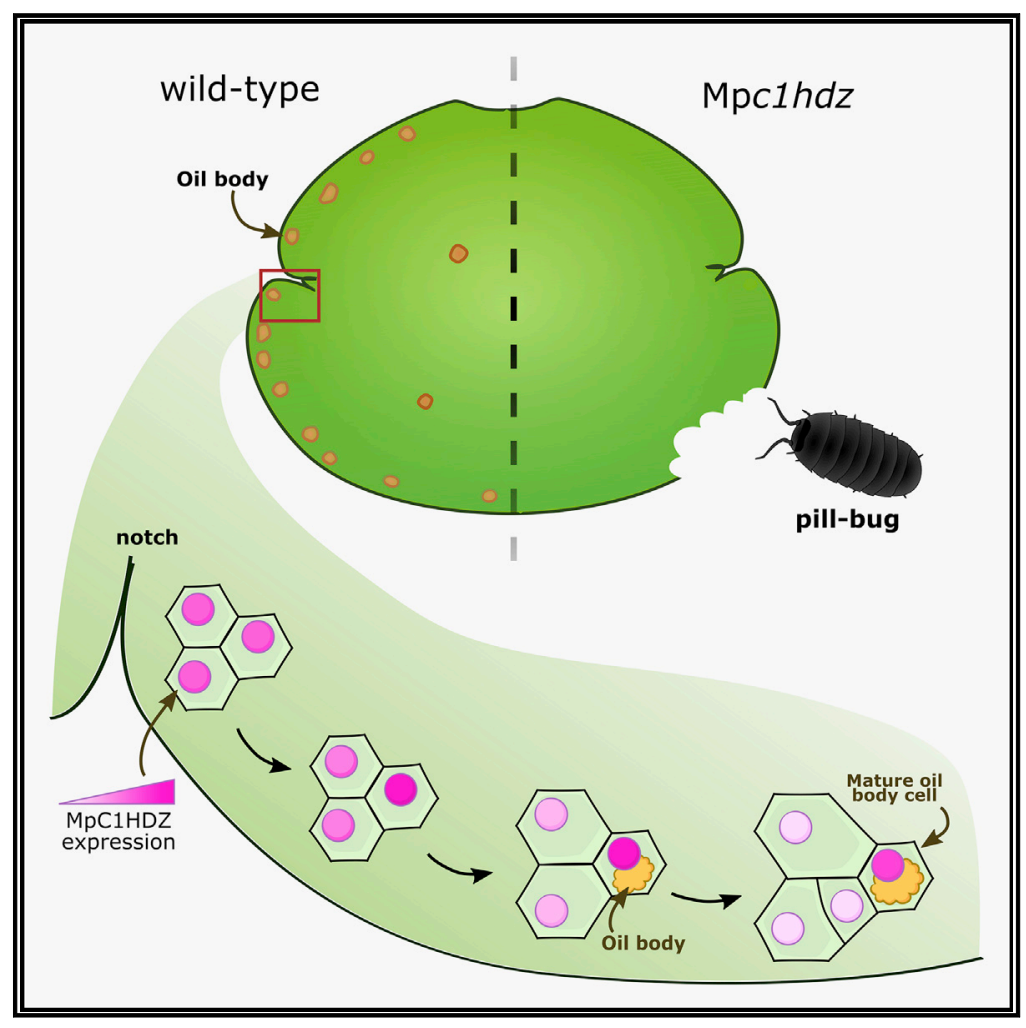

\section{Highlights}

- Oil body cell differentiation in M. polymorpha is positively regulated by $\mathrm{MpC} 1 \mathrm{HDZ}$

- High expression levels of MpC1HDZ precede oil body differentiation

- Terpenoid-rich oil bodies protect the plant against arthropod herbivores

\section{Authors}

Facundo Romani, Elizabeta Banić, Stevie N. Florent, ..., John L. Bowman, Miltos Tsiantis, Javier E. Moreno

\section{Correspondence}

tsiantis@mpipz.mpg.de (M.T.), javier.moreno@santafe-conicet.gov.ar (J.E.M.)

\section{In Brief}

Most of the chemical diversity of liverworts resides within oil body cells, a synapomorphy of the lineage. Romani, Banic et al. show that oil bodies defend the plant against arthropods. Moreover, oil body cell differentiation requires the MpC1HDZ transcription factor, which shows high gene expression levels prior to oil body differentiation. 


\title{
Current Biology
}

\section{Oil Body Formation in Marchantia polymorpha Is Controlled by MpC1HDZ and Serves as a Defense against Arthropod Herbivores}

\author{
Facundo Romani, ${ }^{1,8}$ Elizabeta Banić, ${ }^{2,8}$ Stevie N. Florent, ${ }^{3}$ Takehiko Kanazawa, ${ }^{4}$ Jason Q.D. Goodger, ${ }^{5}$ \\ Remco A. Mentink, ${ }^{6}$ Tom Dierschke, $, 3,7$ Sabine Zachgo, ${ }^{7}$ Takashi Ueda, ${ }^{4}$ John L. Bowman, ${ }^{3}$ Miltos Tsiantis, ${ }^{2,10, *}$ \\ and Javier E. Moreno1,9,11,* \\ ${ }^{1}$ Instituto de Agrobiotecnología del Litoral, Universidad Nacional del Litoral - CONICET, Facultad de Bioquímica y Ciencias Biológicas, Centro \\ Científico Tecnológico CONICET Santa Fe, Colectora Ruta Nacional No. 168 km. 0, Paraje El Pozo, Santa Fe 3000, Argentina \\ ${ }^{2}$ Department of Comparative Development and Genetics, Max Planck Institute for Plant Breeding Research, Carl-von-Linné-Weg 10, 50829 \\ Cologne, Germany \\ ${ }^{3}$ School of Biological Sciences, Monash University, Melbourne, VIC 3800, Australia \\ ${ }^{4}$ Division of Cellular Dynamics, National Institute for Basic Biology, Nishigonaka 38, Myodaiji, Okazaki, Aichi 444-8585, Japan \\ ${ }^{5}$ School of BioSciences, The University of Melbourne, Parkville, VIC 3010, Australia \\ ${ }^{6}$ Bejo Zaden B.V., Warmenhuizen 1749, the Netherlands \\ ${ }^{7}$ Botany Department, School of Biology and Chemistry, Osnabrück University, Barbarastraße 11, 49076 Osnabrück, Germany \\ 8These authors contributed equally \\ 9Twitter: @JaviMorenoOK \\ 10Twitter: @mpipz_cologne \\ ${ }^{11}$ Lead Contact \\ *Correspondence: tsiantis@mpipz.mpg.de (M.T.), javier.moreno@santafe-conicet.gov.ar (J.E.M.) \\ https://doi.org/10.1016/j.cub.2020.05.081
}

\section{SUMMARY}

The origin of a terrestrial flora in the Ordovician required adaptation to novel biotic and abiotic stressors. Oil bodies, a synapomorphy of liverworts, accumulate secondary metabolites, but their function and development are poorly understood. Oil bodies of Marchantia polymorpha develop within specialized cells as one single large organelle. Here, we show that a class I homeodomain leucine-zipper (C1HDZ) transcription factor controls the differentiation of oil body cells in two different ecotypes of the liverwort $M$. polymorpha, a model genetic system for early divergent land plants. In flowering plants, these transcription factors primarily modulate responses to abiotic stress, including drought. However, loss-of-function alleles of the single ortholog gene, MpC1HDZ, in M. polymorpha did not exhibit phenotypes associated with abiotic stress. Rather, Mpc1hdz mutant plants were more susceptible to herbivory, and total plant extracts of the mutant exhibited reduced antibacterial activity. Transcriptomic analysis of the mutant revealed a reduction in expression of genes related to secondary metabolism that was accompanied by a specific depletion of oil body terpenoid compounds. Through timelapse imaging, we observed that MpC1HDZ expression maxima precede oil body formation, indicating that MpC1HDZ mediates differentiation of oil body cells. Our results indicate that $M$. polymorpha oil bodies, and MpC1HDZ, are critical for defense against herbivory, but not for abiotic stress tolerance. Thus, C1HDZ genes were co-opted to regulate separate responses to biotic and abiotic stressors in two distinct land plant lineages.

\section{INTRODUCTION}

The emergence of land flora in the Ordovician and the subsequent radiation of plants into terrestrial environments were major evolutionary events in the history of Earth. This radical breakthrough for life on the planet entailed the evolution of both new developmental programs and biochemical pathways [1]. Early land plants were faced with novel biotic and abiotic stressors, including a desiccating aerial environment, increased solar radiation, and greater interspecific competition, including herbivory. This transition was accompanied by a dramatic increase in the diversity of secondary metabolites and the complexity of transcriptional regulation, to protect plants from herbivores and pathogens, and also abiotic stressors, such as desiccation and light intensity [2-4].

Liverworts represent one of the earliest diverging land plant lineages, with a predicted Ordovician-Silurian origin of all three major extant liverwort clades [5, 6]. One synapomorphy of this lineage is the presence of oil bodies, specialized organelles containing an array of lipophilic secondary compounds [7]. In some liverworts, multiple oil bodies are found in nearly all differentiated cells of both gametophyte and sporophyte generations. Although in other liverworts, such as Marchantia polymorpha (M. polymorpha), oil bodies are large and differentiate in only a subset of isolated cells referred to as idioblasts. They originate in young meristematic cells in a relatively constant proportion [8]. It has long been known 


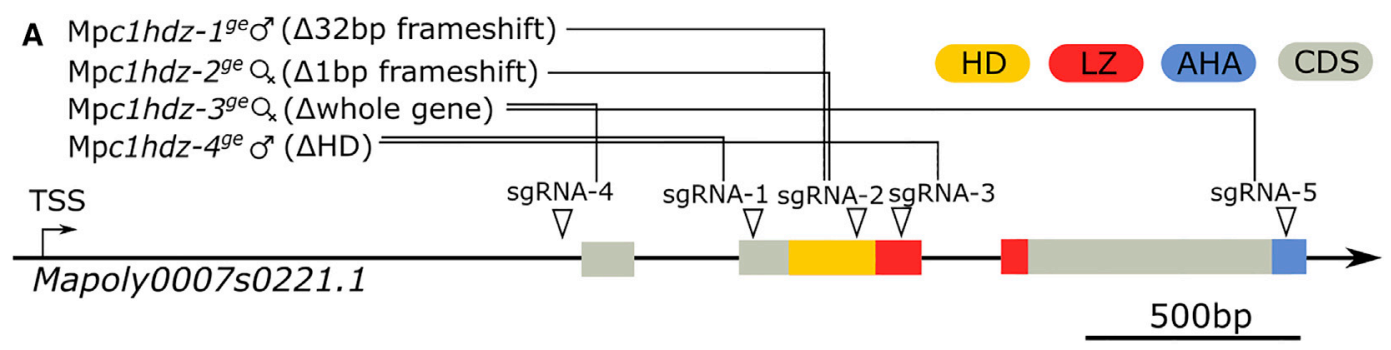

B

WT $\sigma^{x}$

WT $Q_{x}$

Mpc1hdz-1 $1^{g e}$

Mpc1hdz-2ge
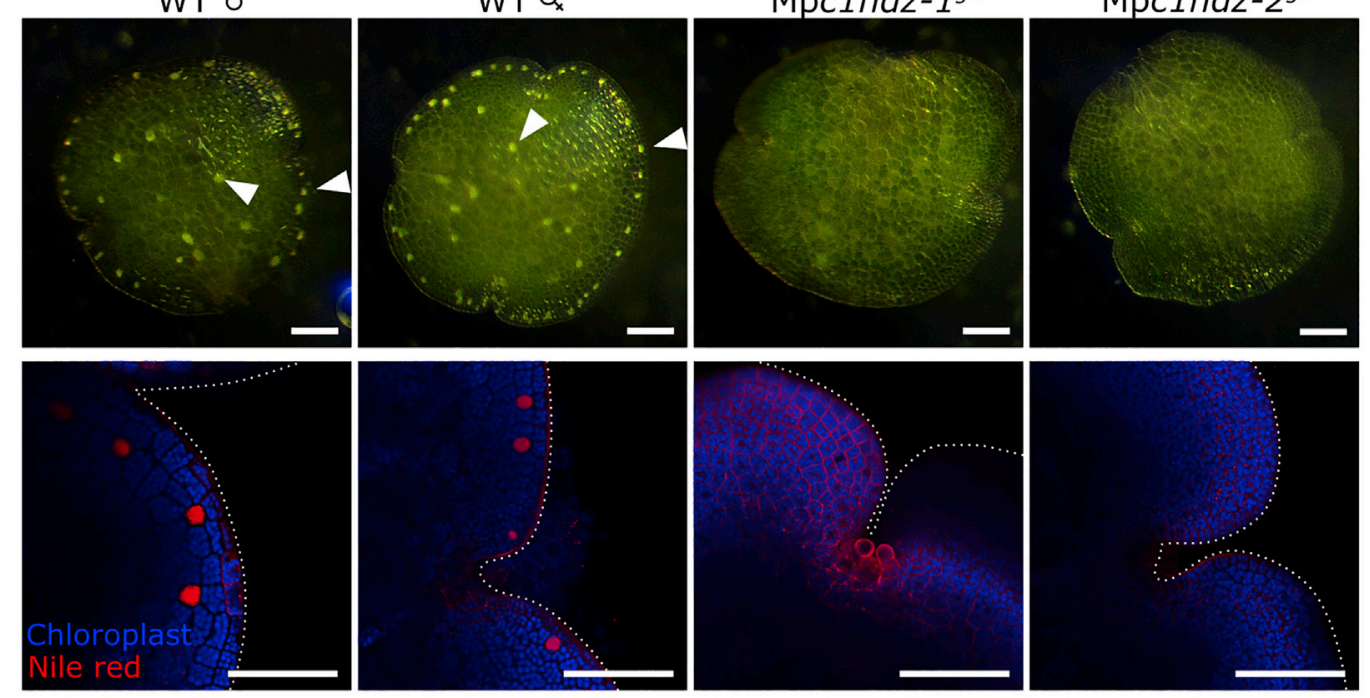

C
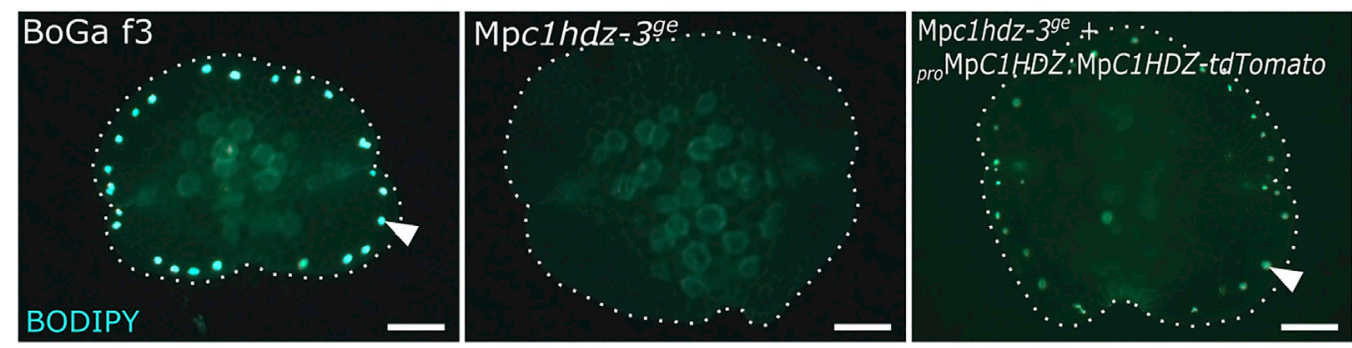

D

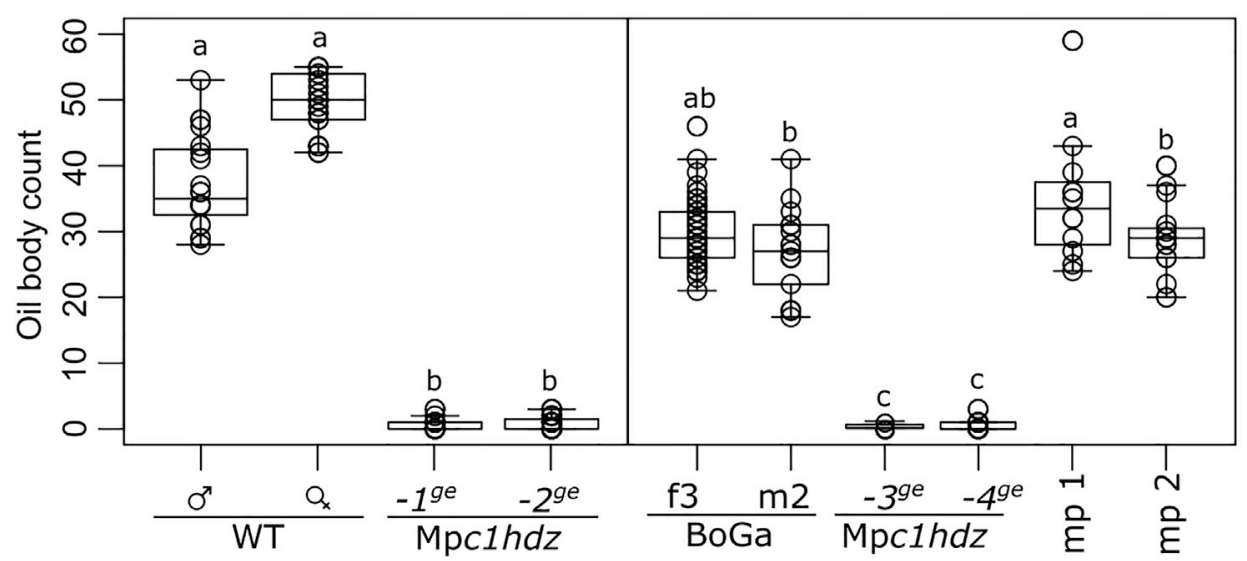

Figure 1. Oil Body Number Is Strongly Reduced in Mpc1hdz Plants

(A) Schematic representation of the MpC1HDZ gene domains and location of designed CRISPR gRNAs targeting the genomic locus (arrowheads). Colored boxes represent the three conserved protein domains. The combination of gRNAs used to generate frameshifts deletions in each case is shown. 
that the liverwort fragrance is associated to the oil body presence [9] and that species-specific differences in taste correlate with oil body chemical composition [10]. Early biochemical analyses identified numerous terpenoids as major chemical constituents of liverwort oil bodies [11, 12], and later studies expanded this list to include diterpenes, bis(bibenzyls), and additional compounds [13-16]. Pfeffer, who gave oil bodies their name (Ölkörper), noted they were membrane bound [17], and later studies confirmed a single membrane [18, 19], in which isoprenoid biosynthesis enzymes are localized [16]. A recent report showed that the oil body membrane results from the fusion of secretory vesicles that requires a cyclic switching of the secretory pathway regulated by MpERF13 transcription factor [20].

Oil body function remains contested, with suggested defensive roles against both abiotic and biotic stressors. Roles protecting against a wide variety of abiotic stressors have been proposed, including cold and osmotic stress [7], desiccation [21-23], high light intensity [24], UV radiation [25, 26], and metabolic stress [18]. In addition, a role against herbivory was postulated by Stahl, who presented snails with fresh or alcoholleached liverworts and noted that snails preferred the latter and did not consume the former to any extent [27]. An anti-herbivory role is also supported by fossilized evidence of herbivore oil body avoidance in Devonian liverworts [28]. Furthermore, the chemical constituents of oil bodies lead to growth inhibition of bacterial and fungal pathogens [29-33]. Despite a large body of literature describing the presence and diversity of oil body secondary metabolites [14, 15, 34, 35], less is known of the regulatory circuits leading to oil body cell differentiation.

Synthesis of specialized metabolites in vascular plants is tightly regulated by transcription factors (TFs), with some orthologs having conserved and others novel roles in liverworts [33, 36-39]. Class I homeodomain leucine-zipper (C1HDZ) TF genes are conserved across streptophytes, with a single homolog predicted to have been present in the common ancestor of land plants [40]. In angiosperms, C1HDZ genes are well-known regulators of tolerance to abiotic stress [41, 42] but also control other molecular functions, including leaf, root, and silique development and vascular patterning [43-45]. However, little is known about the physiological role of C1HDZ in bryophytes [46]. M. polymorpha provides a model to investigate their functional evolution because the genome encodes only one single C1HDZ ortholog (MpC1HDZ) [2]. Here, we investigate $M p C 1 H D Z$ functionality and report that it exhibits a divergent function relative to its angiosperms orthologs.

\section{RESULTS}

MpC1HDZ Controls the Development of Oil Body Cells MpC1HDZ, the solitary C1HDZ M. polymorpha ortholog, possesses all conserved protein motifs [40] defined for $\mathrm{C} 1 \mathrm{HDZ}$ proteins (Figure 1A). Using two different genetic backgrounds (wild type [WT], Australian; BoGa, Botanical Garden Osnabrück, German accession), we targeted guide RNAs (gRNAs) to the HDZ domain generating mutant alleles with premature STOP codons, deletion of the whole HDZ domain, or deletion of the entire locus (Figure 1A). Four lines were clonally purified through gemmae generations: Mpc1hdz-1 ${ }^{\text {ge }}$ (male) and Mpc1hdz-2 ${ }^{g e}$ (female) harbor frameshift mutations that generate early stop codons and were generated in the Australian accession. Mpc1hdz-3 $3^{g e}$ and $\mathrm{Mpc} 1 \mathrm{hdz}-4^{g e}$ were generated in the BoGa accession, deleting the whole CDS or the $\mathrm{HDZ}$ domain, respectively (Figure $1 \mathrm{~A}$; nomenclature as in [47]).

In all cases, mutant plants did not show gross phenotypic defects and completed their life cycle in a similar manner and timing as WT (Figures S1A-S1D). To understand the physiological role of $\mathrm{MpC} 1 \mathrm{HDZ}$, we performed a detailed phenotypic characterization of the mutant alleles. The most conspicuous phenotype of Mpc1hdz mutants is a striking reduction in the number of oil bodies, both in gemmae and thalli, in two different genetic backgrounds (Figures 1B, 1C, S1E, and S1F). M. polymorpha oil body cells are observed as brown dots or highly reflective cells, with 20-50 oil bodies per gemma, mostly located marginally (Figures $1 \mathrm{~B}$ and $1 \mathrm{C}$ ). Whereas mutant gemmae lacked oil bodies, in the thallus and in the parenchymatic region, we found a small number of oil bodies in the tissue (Figures S1E-S1G). It is known that specific environmental conditions, such as starvation and field non-axenic cultivation, affect oil body cell number [15]. WT plants grown in Petri dishes developed fewer oil body cells compared to non-axenic grown plants and starvation-treated plants (Figure S1E). In contrast, the number of oil body cells of the Mpc1hdz mutant remained low in both conditions (Figure S1E).

To examine oil body distribution throughout the life cycle, we crossed Mpc1hdz-2 ${ }^{g e}$ with Mpc1hdz-1 ${ }^{g e}$. The resultant sporophyte-produced spores of the two genotypes and sporelings were phenotypically WT. After 18 days, oil bodies were visible in WT sporelings but were totally absent in Mpc1hdz sporelings (Figure S1F). Thus, we conclude that MpC1HDZ is a positive regulator of oil body formation across all organs and developmental stages tested.

To confirm the role of MpC1HDZ in the oil body phenotype, we complemented the mutant using the endogenous promoter of $\mathrm{MpC1HDZ}$ driving MpC1HDZ-tdTomato (proMpC1HDZ $\left.{ }^{3 k b}: \mathrm{MpC} 1 \mathrm{HDZ}-t d T o m a t o\right)$ in the BoGa ecotype, which rescued the oil body phenotype (Figures $1 \mathrm{C}$ and 1D). In contrast, when $\mathrm{MpC} 1 \mathrm{HDZ}$ expression was driven by either of two constitutive promoters (proEF1:MpC1HDZ, pro35S:MpC1HDZ), no viable plants were obtained, suggesting that constitutive overexpression is lethal (data not shown).

(B) Phenotype of freshly collected gemmae from WT and Mpc1hdz mutant plants under light microscopy (top panel); confocal microscopy of Red-Nile-stained gemmae (bottom panel). Red Nile fluorescence is shown in red and chloroplast auto-fluorescence in blue.

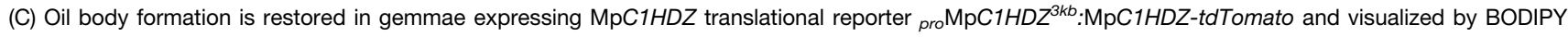
staining.

(D) Number of oil bodies per gemma in WT, mutant, and complemented plants in both genetic backgrounds were quantified and represented as boxplot ( $\mathrm{n}>12$ ).

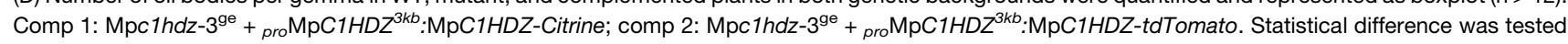
using ANOVA followed by Tukey HSD $p<0.01$; letters indicate statistically significant groups.

See also Figure S1. 


\section{$\infty$ CellPress}

\section{Current Biology

The MpC1HDZ Promoter Is Active in Thallus and Gemmalings with High Expression in Oil Body Cells To better understand the relationship between $\mathrm{MpC} 1 \mathrm{HDZ}$ and oil body cells, we studied the gene expression pattern with transcriptional reporters (oro MpC1HDZ:GUS) using the regulatory region of each genetic background. Strong GUS activity was observed in oil body cells in gemmae (Figure 2A). At later stages, GUS activity was also detected in the center of the thallus and in meristematic regions (Figures $2 \mathrm{~B}$ and $2 \mathrm{C}$ ), at the notch extending into the midrib, including the gemma cups (Figures 2C-2E), and broadly in reproductive organs (Figure $2 \mathrm{~F}$ ). A similar transcriptional reporter activity was also observed in the BoGa ecotype (Figures S2A-S2F). We searched for the presence of potential cis-regulatory elements in the promoter region using PlantRegMap [48]. The analysis revealed multiple binding sites ranging from 8 to $30 \mathrm{bp}$ length. Most notable was the presence of ERF and TCP binding sites, and other binding sites, including BBRBPC, MYB, LBD, basic-helix-loop-helix (bHLH), and NAC (Table S1), are also present.

To further test the role of MpC1HDZ in responses to environmental conditions, we used the transcriptional reporter in a wide range of stressors (Figure S5A). Unlike in angiosperms [41], MpC1HDZ was not induced by abiotic stress treatments or abscisic acid (ABA) (Figure S5A). However, among environmental conditions known to induce oil bodies, we found a strong induction of $\mathrm{MpC} 1 \mathrm{HDZ}$ expression in the meristematic region when plants were grown in non-axenic conditions (soil pots), but not in starvation (Figure S5A).

\section{MpC1HDZ Expression Maxima Precede Oil Body} Formation

To understand the dynamics of oil body formation and MpC1HDZ expression, we used a translational reporter proMpC1HDZ ${ }^{3 k b}: \mathrm{MpC} 1 \mathrm{HDZ}$-tdTomato that can complement the mutant phenotype. A fluorescently labeled MpC1HDZ-tdTomato was nuclear localized in stable $M$. polymorpha transgenic lines (Figure 2G). In 3-day-old gemmalings, high-expressing cells were located close to the notch in undifferentiated meristematic cells (Figures 2G and S2G-S2J). MpC1HDZ-tdTomato expression levels dropped gradually from the apical notch to distant cells, except for some isolated cells where MpC1HDZtdTomato accumulation became markedly higher compared to neighboring cells (Figure 2G). Using confocal microscopy to quantify the fluorescence of MpC1HDZ-tdTomato and oil bodies stained using BODIPY 493/503 dye, we found an association between MpC1HDZ-tdTomato accumulation and oil body cells (Figure 2H). Closer to the apical notch, sporadic cells strongly expressed MpC1HDZ-tdTomato without BODIPY signal in their vicinity. However, in cells more distant from the apical notch, the brighter MpC1HDZ-tdTomato nuclear signal was associated with BODIPY-stained oil bodies (Figures $2 \mathrm{G}$ and $2 \mathrm{H}$ ). This initial correlation was further confirmed with cell-tracking experiments. We observed that $11 / 13$ newly detectable oil bodies in the field of view formed in cells that had exceptionally high MpC1HDZ-tdTomato expression in a previous time point (Figures 2I, 2J, and S4). These results suggest that $\mathrm{MpC} 1 \mathrm{HDZ}$ expression maxima precede oil body formation. Taken together, our results indicate that oil body cell differentiation is positively regulated by the MpC1HDZ.

\section{Mpc1hdz Mutants Show Normal Abiotic Stress}

Responses

Ectopic expression of C1HDZ genes from different angiosperms in Arabidopsis enhances tolerance to water stress, such as water deprivation, osmotic stress, and salt stress [41, 49-53]. Moreover, some Arabidopsis loss-of-function C1HDZ alleles showed a reduced sensitivity to $A B A$ treatments, consistent with a role in water stress tolerance [54, 55]. Using established protocols to manipulate abiotic stress conditions, we applied different treatments, including water deprivation and osmotic and salt stress, to quantify the impact on plant growth, i.e., area growth and thallus biomass. Our results showed no altered growth responses of the mutant lines compared to the WT (Figures 3A-3C and S6). We also performed a water-loss experiment of detached thalli and an ABA-dose response germination assay. We did not find a differential response of the mutant in either case (Figures 3D and 3E). The Marchantia HDZ family comprises the four canonical classes found in land plants with only one gene member per class [40]. Because HDZ genes of different classes respond to abiotic stress, we analyzed the expression landscape of $\mathrm{MpC} 1 \mathrm{HDZ}$ and the other $\mathrm{HDZ}$ genes under abiotic stress. To this end, we performed qRT-PCR analysis under $\mathrm{NaCl}$ and ABA treatments at two time points. Unlike the strong induction observed for $M p D H N 1$, a marker gene of the Marchantia abiotic response [56], MpHDZ expression remained unaltered or was slightly reduced under these experimental conditions (Figures S5).

Oil bodies have long been proposed to protect liverworts against other abiotic stresses [57-59]. Therefore, we examined the response of $\mathrm{Mpc} 1 \mathrm{hdz}$ plants to a wide range of other abiotic stressors. We grew plants in axenic control conditions for 1 week and subsequently transferred plants to different stress environments and measured growth parameters under cold stress, high light irradiance, and UV light treatments (Figure S6). We also measured growth parameters in plants subjected to treatments that promote the formation of oil bodies (Figure S6). We did not observe significant differences in growth parameters among WT and Mpc1hdz plants in any of the tested conditions. Overall, our results suggest that neither oil bodies nor MpC1HDZ are involved in the abiotic stress responses of $M$. polymorpha and that C1HDZ gene function observed in angiosperms is not conserved throughout land plants.

\section{Mpc1hdz Mutants Have an Impaired Biotic Defense Response}

Because oil bodies are rich sesquiterpenes stores and these compounds are known to play a central role in the ecology of plant-biotic interactions, together with old experiments associating the presence of oil bodies to the feeding behavior of snails [27], we investigated whether oil bodies play a role in the biotic response of $M$. polymorpha. We performed a choice experiment using WT and Mpc1hdz plants to the terrestrial isopod Armadillidium vulgare (pill bug; Crustacea). In this case, we monitored the total thalli area consumed by starved pill bugs for $24 \mathrm{~h}$. The total area lost from mutant alleles was significantly larger compared to respective WT plants in both genetic ecotypes (Figures $4 A-4 C$, S7A, and S7B). Thus, isopods prefer mutant plants over WT, consistent with the possible role of oil bodies as a defense against arthropods herbivory. 

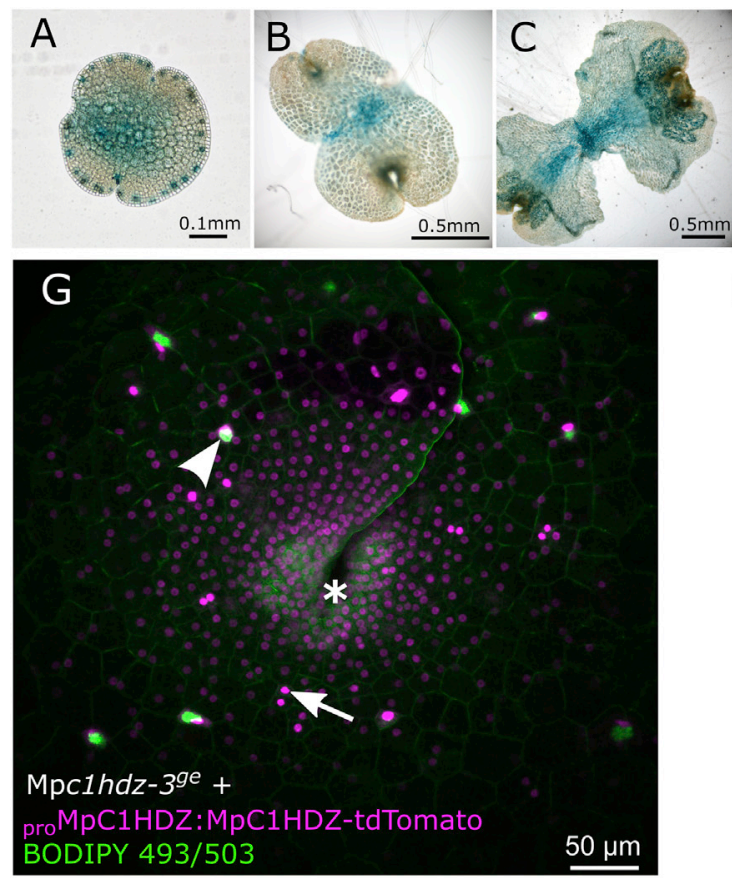

$\mathrm{H}$
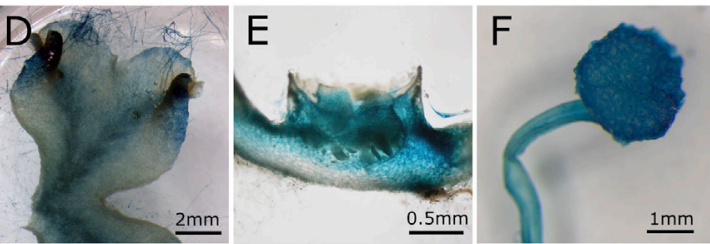

$\mathrm{H}$

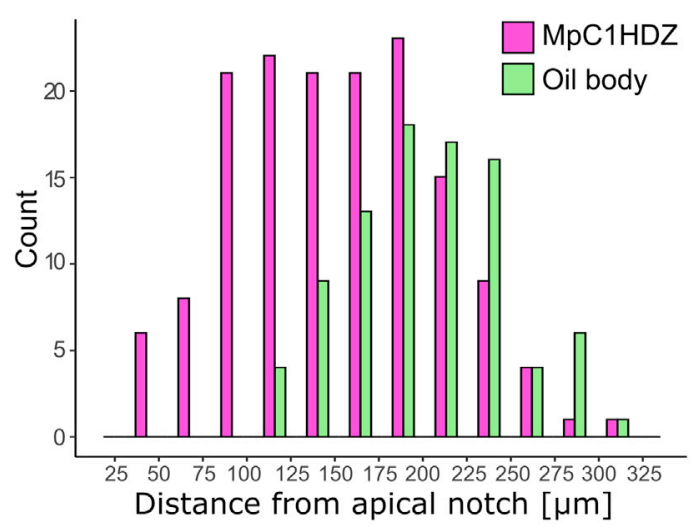

Figure 2. Strong MpC1HDZ Expression Precedes Oil Body Formation

(A-F) Representative pictures taken with light microscopy of proMpC1HDZ ${ }^{4.6 k b}$ :GUS staining (from left to right) of (A) gemma, (B) 3-day-old gemmaling, (C) 1-week-old gemmaling, (D) 3-week-old plant, (E) transverse section of gemma cup, and (F) young antheridiophore of Australian ecotype plants grown in nonaxenic conditions.

(G) A representative apical notch area of Mpc1hdz-3 ${ }^{g e}$ gemmaling complemented with pro MpC1HDZ ${ }^{3 k b}$ :MpC1HDZ-tdTomato and stained with BODIPY 493/503. Sum slices projection of a confocal stack shows pro MpC1HDZ ${ }^{3 k b}: M p C 1 H D Z$-tdTomato signal in magenta and BODIPY in green. Asterisk marks the apical notch, arrowhead marks an oil body, and arrowhead marks an exceptionally bright nucleus.

$(\mathrm{H})$ Distribution of MpC1HDZ-tdTomato expression maxima and oil body distances from apical notch in 3-day-old gemmalings, quantified from 2D projections as in (G). Quantification method is described in Figure S3. All bright nuclei above a defined MpC1HDZ-tdTomato fluorescence intensity threshold and all oil bodies from 11 replicates were counted. Significance was tested using Wilcoxon rank-sum test; $p<0.05$.

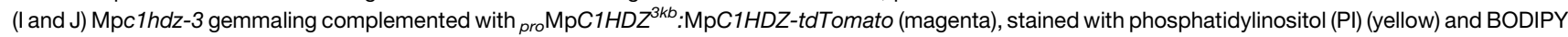
493/503 (green) and observed using CLSM microscopy. Representative pictures at $t_{0}(\mathrm{l})$ and $t_{1}(\mathrm{~J})$ time points of the same gemmaling region in $12 \mathrm{~h}$ intervals (standard deviation z stack projections). Asterisks of the same color mark the corresponding landmarks in different time points. The blue asterisks indicate an air pore. Arrow points to nucleus of the cell that developed an oil body (arrowhead) within the next $12 \mathrm{~h}$. All 13 observed transition events are shown in Figure S4, and the event shown here corresponds to number 6 (Figures S4I and S4J). Scale bars are shown in the picture.

See also Figures S2-S4 and Table S1. 

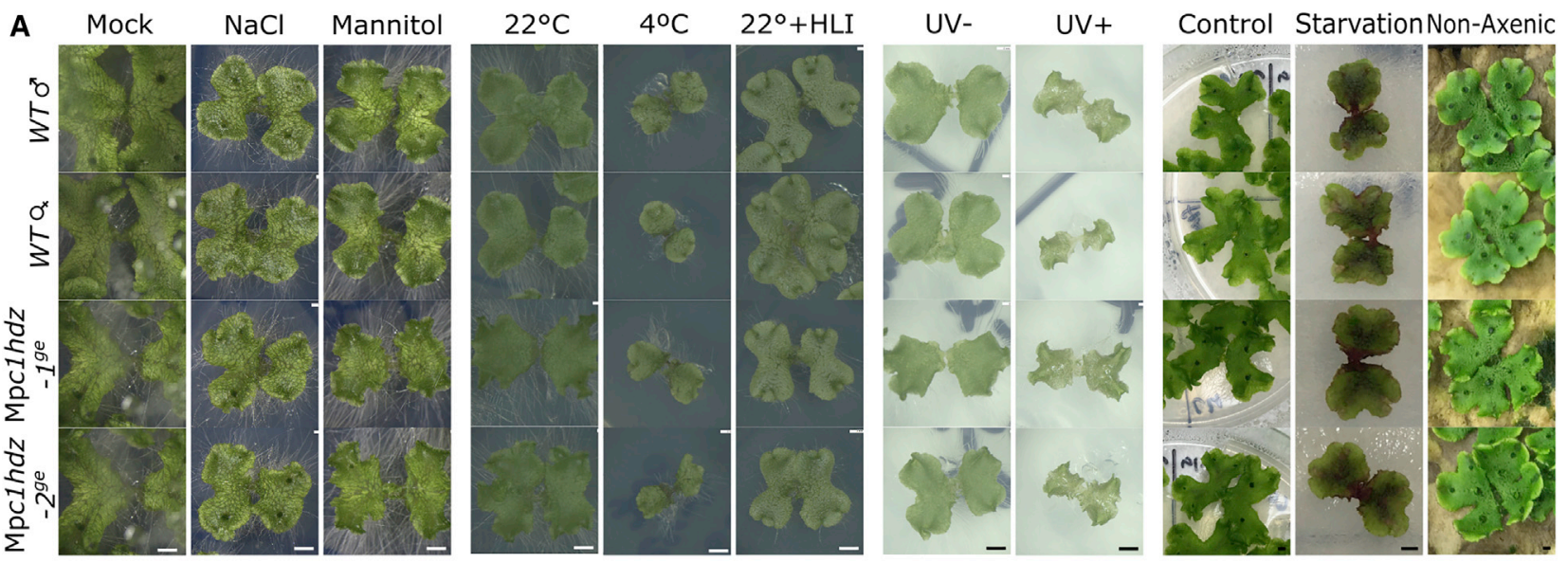

\section{B Osmotic stress}

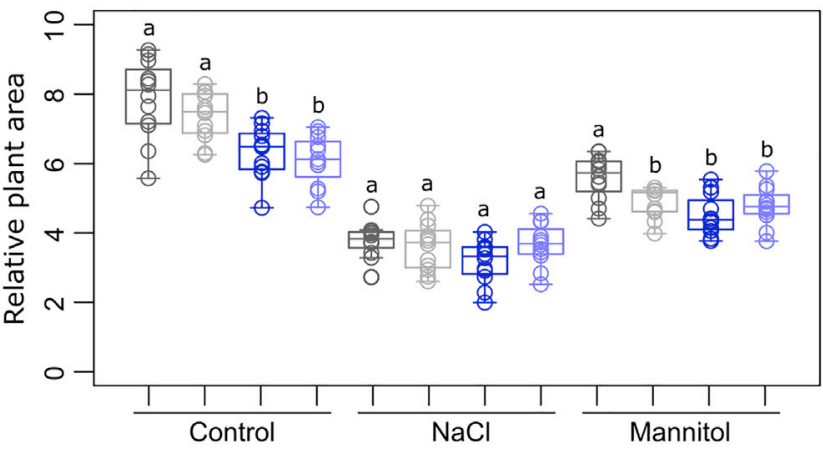

C

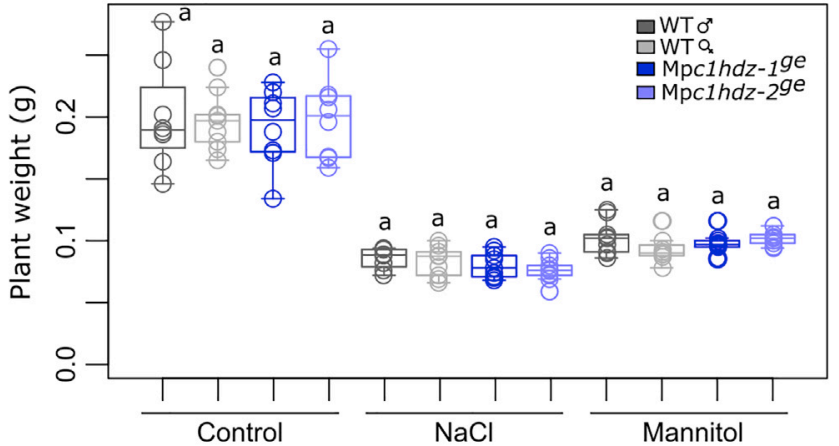

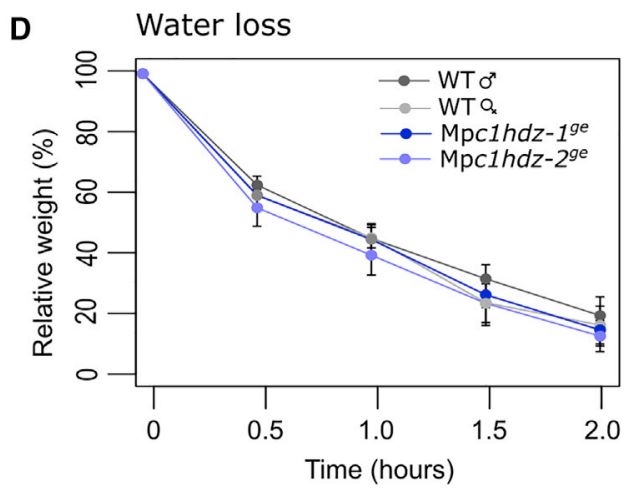

E ABA-dose response germination
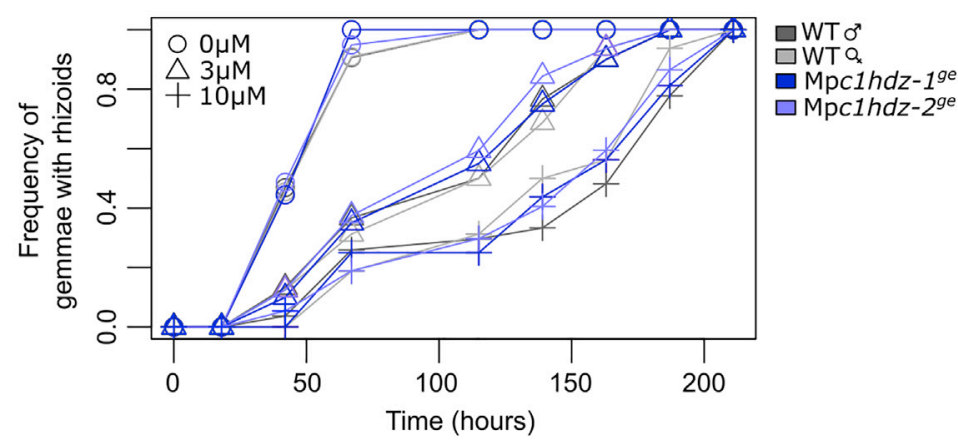

Figure 3. Mpc1hdz Plants Have an Intact Growth Response to Abiotic Stress

(A) Representative picture of phenotypic effect of abiotic stress treatments on Mpc1hdz and WT plants as explained in STAR Methods section. Treatments are indicated at the top of illustrative pictures. Scale bars $(2 \mathrm{~mm})$ are indicated in the bottom panels. Individual values are indicated as circles.

(B-D) Quantification of exposed area (B) and weight (C) of 3-week-old plants under osmotic stress treatments. Individual values are indicated as circles. Full quantifications for each treatment are shown in Figure S6.

(D) Water loss experiments were done using 3-week-old WT and Mpc1hdz plants placed on filter paper towel and weighed every 30 min for $2 \mathrm{~h}$ at room temperature. Bars show the average of three biological replicates \pm SE of the mean. No statistical difference was observed using ANOVA followed by Tukey HSD after $1 \mathrm{~h}$ of treatment $(\mathrm{p}>0.05)$.

(E) Gemmae dormancy under ABA treatments at different concentrations and time points (left, $\mathrm{n}=\sim 25$ ). No statistical difference was found testing equal frequencies against WT using exact binomial test $(p>0.05)$ at $115 \mathrm{~h}$.

See also Figures S5 and S6.

As several compounds produced in oil bodies also have antibiotic activity [33,60], they may provide further protection against biotic assault. To test this, we applied ethanolic extracts dissolved in DMSO from WT and Mpc1hdz thalli to the Gram (+) soil bacteria, Bacillus subtilis, using an overlay assay. We expected that WT extracts might inhibit bacterial growth to a larger extent than mutant plants extracts containing reduced levels of terpenoid and bis(bibenzyl) compounds. We observed that antibacterial activity was reduced in 
Pill-bug feeding assay

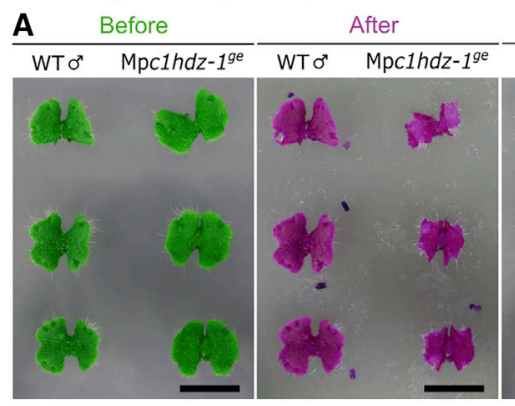

C

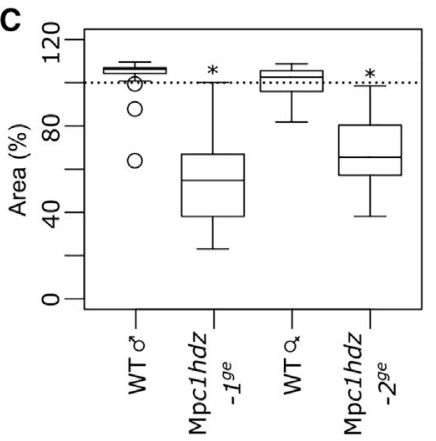

WT $\sigma^{\text {M }}$ Mpc1hdz-1 ${ }^{g e}$

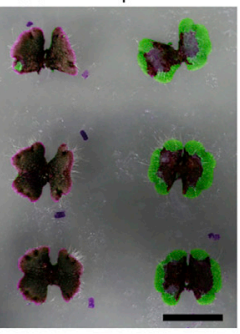

D

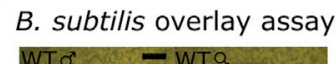

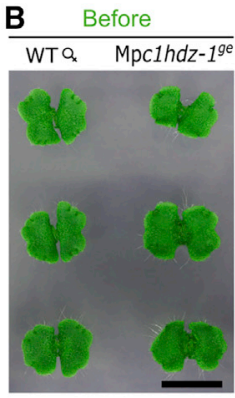
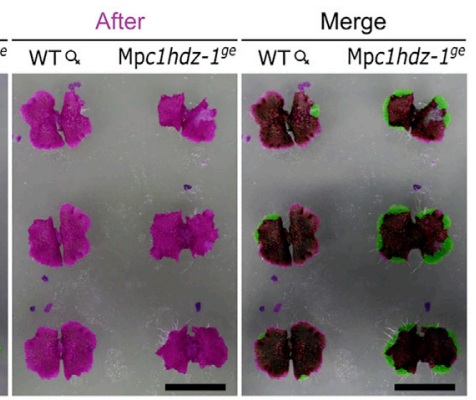

E
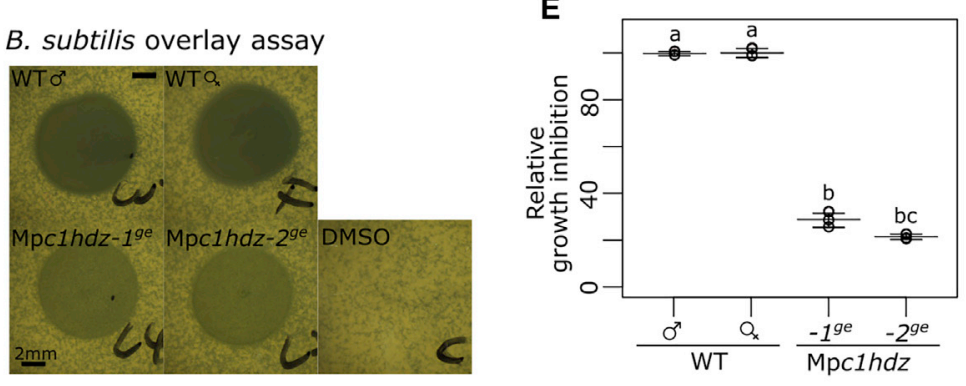

Figure 4. Phenotypic Analysis of Mpc1hdz Plants Challenged with Biotic Stressors

(A and B) Feeding assay was performed using starved pill bugs (Armadillidium vulgare), and 10-day-old thalli were co-cultivated for $24 \mathrm{~h}$. In order to contrast thallus areas, plants were colored in green (before) and magenta (after). Male WT and Mpc1hdz-1 $1^{\text {ge }}$ thalli (A) are shown. Female WT and Mpc1hdz-2 ${ }^{g e}$ thalli (B) are shown. Scale bars represent $1 \mathrm{~cm}$.

(C) Thallus damage quantification was performed using ImageJ and data represented as boxplots. Thallus area change indicates the ratio of thallus area after and before $(n=36)$. Statistical differences are marked as asterisks and computed pairwise $t$ test $(p<0.01)$ comparing each mutant with their respective control.

(D) Bacillus subtilis overlay assay using ethanolic lipid extracts from WT and Mpc1hdz mutant thalli dissolved in DMSO. Representative images of experimental results are shown (three replicates were performed with consistent results). Scale bars represent $2 \mathrm{~mm}$.

(E) Quantification of relative growth inhibition as density of colonies relative to WT. The graph shows the mean with individual values $(\mathrm{n}=3$ ). Statistical difference was tested using ANOVA followed by Tukey HSD p $<0.01$; letters indicate statistically significant groups.

See also Figure S7.

Mpc1hdz extracts in a dose-dependent manner, around one order of magnitude compared to WT extracts (Figures 4D, 4E, and S7C). Thus, in contrast to abiotic stresses, the striking phenotypic effects observed when challenged with biotic stressors provide convincing evidence that oil bodies are critical for biotic defense responses.

\section{MpC1HDZ Alters the Expression of Terpenoid-Related \\ Genes}

To better understand the molecular mechanisms underlying the altered defense response of the Mpc1hdz mutant, we compared the genome-wide transcript profiles of WT and Mpc1hdz plants grown in control (axenic) and non-axenic conditions. Here, we show the results of consistently differentially expressed genes between both RNA sequencing (RNA-seq) experiments (Figures 5A and $5 \mathrm{~B})$. In control conditions, we identified 217 downregulated genes $\left(\log _{2}(\mathrm{FC})<-1\right.$; adj. $\left.\mathrm{p}<0.05\right)$ and 131 upregulated genes $\left(\log _{2}(\mathrm{FC})>1\right.$; adj. $\left.\mathrm{p}<0.05\right)$ in Mpc1hdz mutant plants (Figure 5), whereas, in non-axenic conditions, we identified 602 genes downregulated and 267 upregulated (Figure 5). Additionally, we performed enrichment analysis over differentially expressed gene groups using an in-house pipeline of automatically annotated gene lists, including GO terms, biological processes, and protein families (Table S2). Among downregulated genes, there was an enrichment in biological processes associated with secondary metabolism. Moreover, we found enrichment in proteins associated with terpene synthases (TPSs) (Table S2). Among upregulated genes, we did not find any significant enrichment.

Within downregulated genes associated with secondary metabolism (Figure 5A) and downregulated in both experiments, we found five MpTPSs (Figure 5A), including mono- and di-TPS with a bacterial, fungal, or plant origin [34, 61]. We also detected other genes involved in the biosynthesis of terpene precursors, including a deoxyxylulose 5-phosphate synthase (Mp4g14720, $\mathrm{MpDXS}$ ) and a cis-prenyltransferase (Mp3g18510, MpCPT5) gene (Figure $5 \mathrm{~A}$ ). In addition, three TF genes (Mp3g07510, MpMYB02; Mp5g01530, MpNAC06; and Mp2g16340, MpTrihelix28) are downregulated (Figure 5A). MpMYB02 is known to regulate cyclic bis(bibenzyl) acids biosynthesis [36], which accumulates in oil bodies [15]. The mutant also exhibited significantly reduced expression of MpSYP12B (Mp4g20670), a N-ethylmaleimide-sensitive factor attachment protein receptor (SNARE) protein with specific localization at the oil body membrane of M. polymorpha [62]. Finally, we also identified other downregulated genes that might be associated with the secondary metabolism, including a catechol-O-methyltransferase (Mp5g06850, MpCOMT), two dirigent proteins (Mp3g24140, MpDIR48 and Mp3g24210, MpDIR60), an ABC transporter (Mp8g13070, $\mathrm{MpABCG1)}$, and cytochrome $\mathrm{P} 450$ coding genes. 
A

Down-regulated in Mpc1hdz

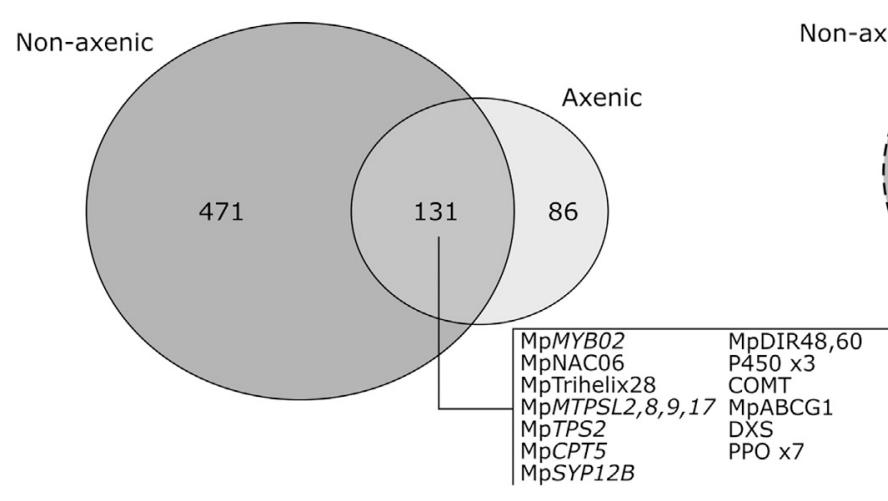

B Up-regulated in Mpc1hdz

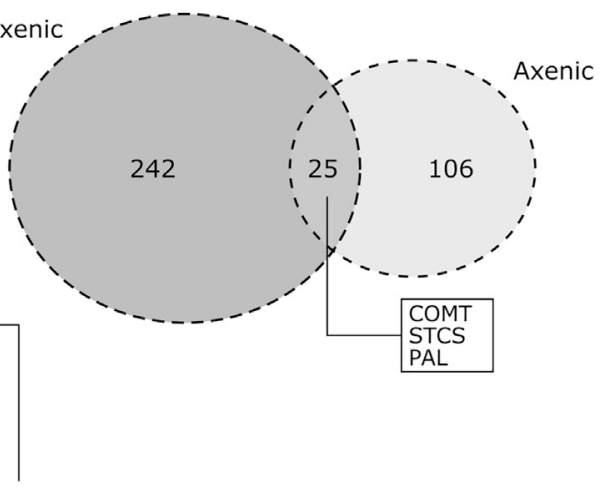

Figure 5. Transcriptomic Analysis of Mpc1hdz Mutants

Venn diagrams of group of genes downregulated $\left(A ; \log _{2}(F C)<-1\right.$; adj. $\left.p<0.05\right)$ and upregulated $(B ; \log 2(F C)>1$; adj. $p<0.05)$ in 3 -week-old vegetative thalli of Mpc1hdz compared to WT in control (axenic) and non-axenic conditions as described in the STAR Methods. Manually annotated genes associated with TFs, terpenoid-related biosynthesis, and other secondary metabolite biosynthetic processes are highlighted. See also Tables S1, S2, and S4.

\section{Oil-Body-Specific Terpenoids Are Depleted in Mpc1hdz} Mutant Plants

The strong reduction of oil bodies and the low expression of some MpTPS genes and other genes related to secondary metabolism led us to explore the terpenoid profile of $\mathrm{Mpc} 1 \mathrm{hd} z$ vegetative thalli. With the aim of evaluating the impact of environmental conditions on oil body chemistry, we also compared the terpenoid profile of plants grown under control, non-axenic, and starvation conditions (Figure 6). In agreement with previous results [15], gas chromatography with flame ionization detector (GC-FID) and gas chromatography-mass spectrometry (GC-MS) analyses of thalli hexane extracts showed that non-axenic growth and starvation significantly increased the amount of terpenoid-related compounds compared to WT backgrounds, with starvation the most inductive condition (Figure 6). Interestingly, male backgrounds showed higher levels of terpenoids than female ones, even in the mutant alleles (Figure 6; Data S1).

More importantly, the chemical profile of Mpc1hdz thalli revealed a specific decrease of terpenoid-related compounds (27 ), such as cis-thujopsene, $\beta$-chamigrene, $\beta$-himachalene, and 5-hydroxy-gurjunene (Figures $6 \mathrm{~A}$ and 6B). Most compounds that accumulated under oil-body-inducing conditions in WT plants were concomitantly reduced in mutant plants, suggesting they are located in oil bodies as suggested previously [15, 16] (Figures 6A and $6 \mathrm{~B})$. The most abundant monoterpene d-limonene (1) and the unidentified terpenoid U13 (10; Figures 6C and 6E) [34] were strongly depleted in the mutants, but they accumulated to similar levels in control and non-axenic conditions (Figure 6C). In contrast, the most abundant diterpene, phytol (11; Figure 6D) [34], fatty acid compounds (8, 9, 12, and 13; Figure 6E), and phytosterols, also present in plant extracts, did not significantly change in the mutant or among growth conditions (Data S1). Taken together, these results support that oil-body-localized terpenoid biosynthesis is affected in Mpc1hdz mutants.

\section{DISCUSSION}

C1HDZ genes have been previously characterized for their roles during abiotic stress response in angiosperms, more specifically to water stress tolerance in Arabidopsis, coffee, cotton, rice, and sunflower [41]. Likely due to functional redundancy in angiosperms, the majority of C1HDZ mutants in angiosperms exhibit subtle phenotypes [40], with some exceptions. Homologs of LATE MERISTEM IDENTITY 1/REDUCED COMPLEXITY alter leaf morphology via local growth repression at early developmental stages [63, 64]. Given that $M$. polymorpha possesses a single $C 1 H D Z$ gene, this is the first time that a full loss-of-function mutant is generated for this subfamily. Interestingly, the lack of a functional $\mathrm{MpC} 1 \mathrm{HDZ}$ did not generate major pleiotropic effects as observed for other TF families in M. polymorpha. Using a wide array of experimental setups, we found no evidence supporting a physiological role for $\mathrm{MpC} 1 \mathrm{HDZ}$ in the abiotic stress response of $M$. polymorpha (Figures 3 and S6). Further, in the moss Physcomitrella patens, only 1 out of $17 \mathrm{C} 1 \mathrm{HDZ}$ genes responded to dehydration and mannitol treatments, and the only studied Ppc1hdz mutant presented a divergent function [46, 65]. These data suggest that the physiological role of $\mathrm{C} 1 \mathrm{HDZ}$ genes in response to abiotic stress may be largely a vascular plant phenomenon.

\section{MpC1HDZ-Mediated Depletion of Oil Bodies Affects Arthropod Herbivory}

We found compelling evidence that $\mathrm{MpC} 1 \mathrm{HDZ}$ modulates, directly or indirectly, the differentiation of oil body cells. Consistently, loss-of-function Mpc1hdz alleles in two ecotypes exhibited a strong reduction in the number of oil bodies (Figure 1). Early experiments showed that the number and size of oil bodies in liverworts remained mostly unaffected in a variety of environmental conditions, such as light, temperature, and limiting nitrogen or phosphorus [8,66,67], and that, once formed, they can be stable in herbarium specimens [10]. It was recently shown, however, that axenic growth resulted in a significant reduction in oil body cell formation, with a substantial induction upon a shift to non-axenic growth, such as cultivation on soil substrate where liverworts are challenged with biotic stressors [15]. We found that $\mathrm{Mpc} 1 \mathrm{hdz}$ plants exhibit a severe reduction of oil body formation under all tested environmental conditions. However, the presence of a limited number of oil bodies in the mutants 

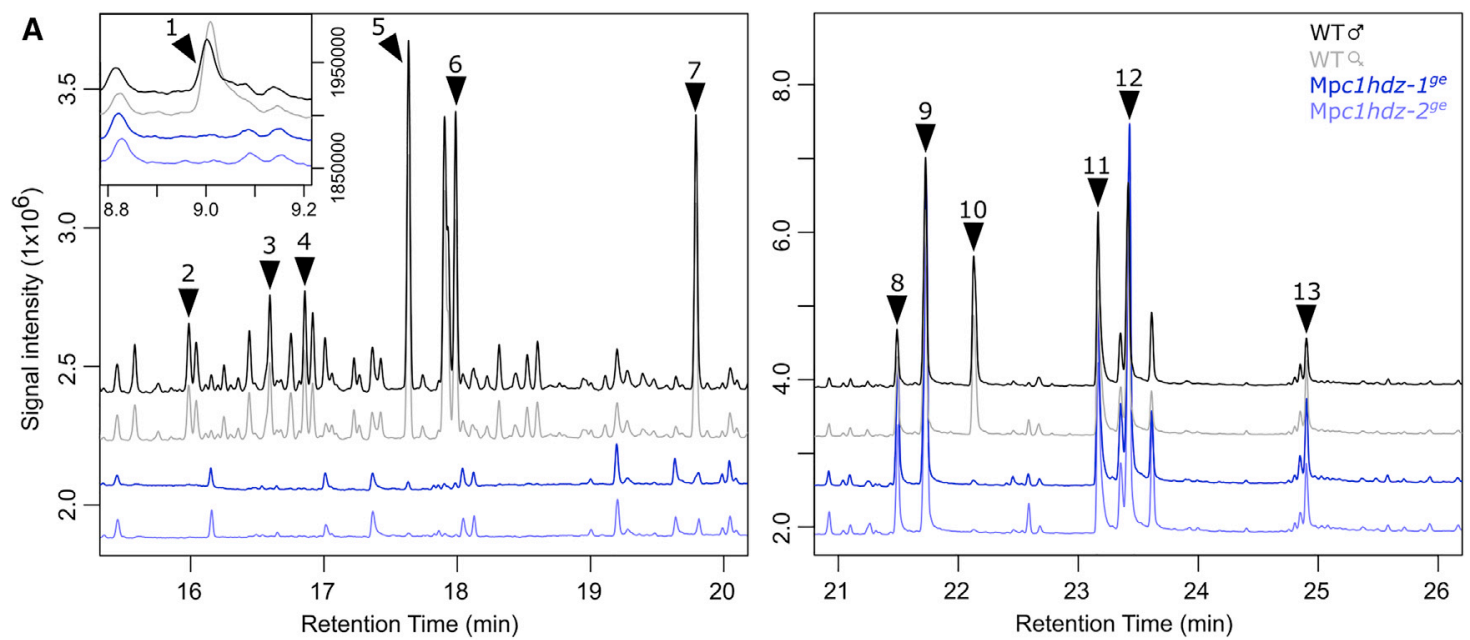

WT a

WT 0 Mpc1hdz-1

B
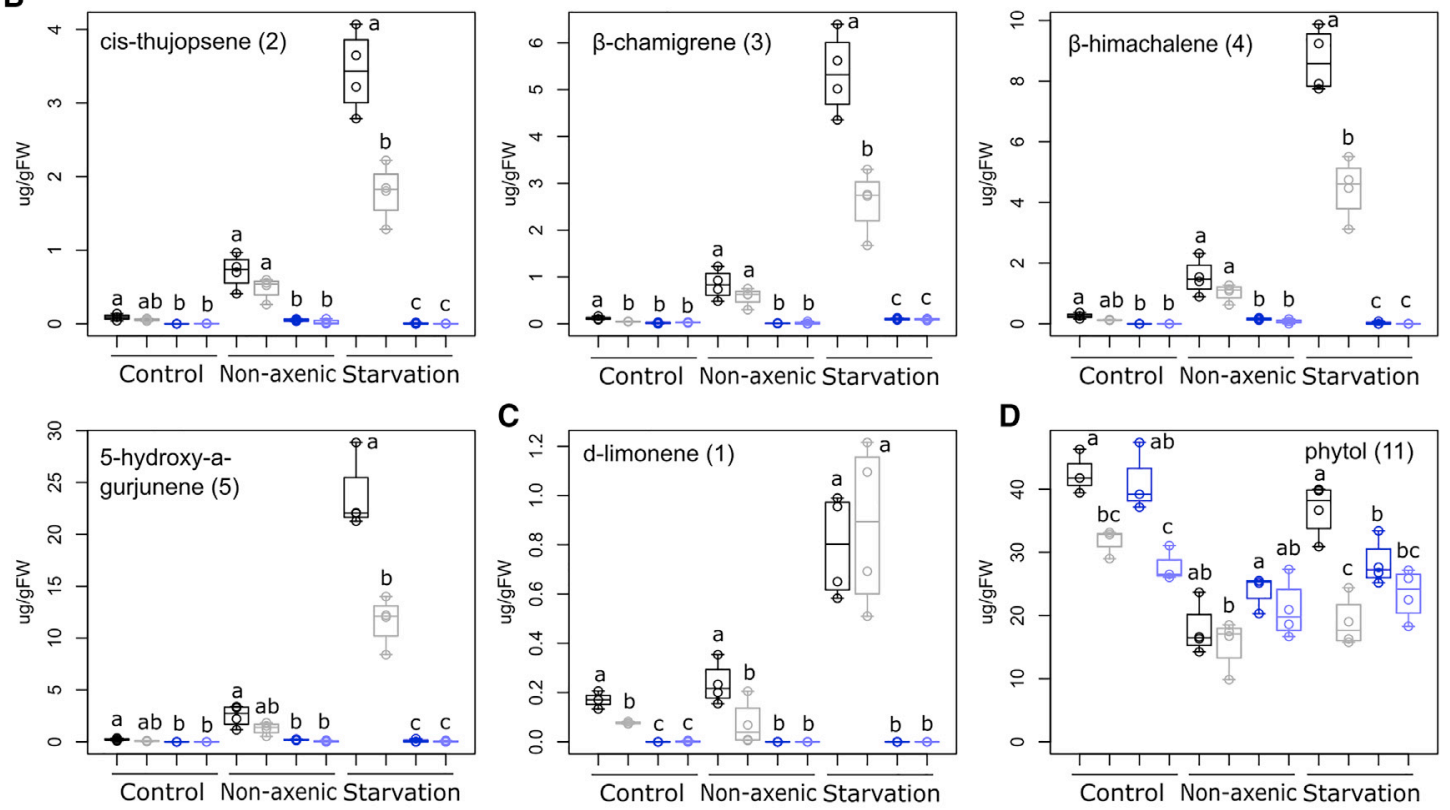

C
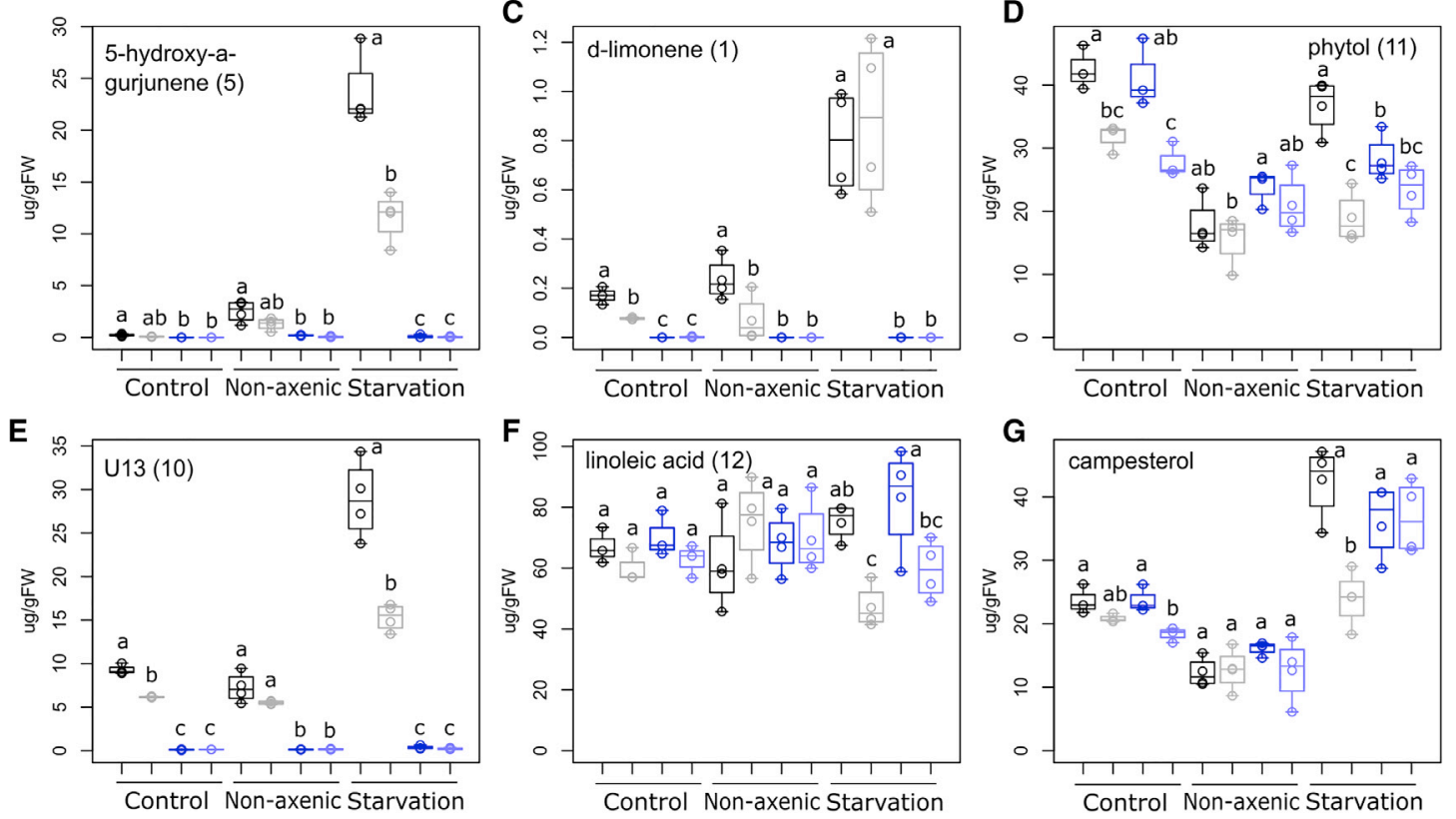

Figure 6. GC-FID Analysis of Mpc1hdz Mutants

(A) Representative GC-FID chromatogram obtained from hexane extracts under starvation treatment of Mpc1hdz and WT plants. Three different ranges are shown. Arrow points represents metabolites: limonene (1); cis-thujopsene (2); $\beta$-chamigrene (3); $\beta$-himachalene (4); 5 -hydroxy-gurjunene (5); viridiflorol (6); cyclocolorenone (7); stearic acid methyl ester (8); palmitic acid (9); unidentified terpenoid U13 (10); phytol (11); linoleic acid (12); and timnodonic acid (13). (B-E) Quantification of representative major metabolites in Mpc1hdz and WT plants under three different treatments as described before (control, non-axenic, and starvation) and represented as boxplots $(n=3-4)$ : total sesquiterpenes $(B)$; monoterpenes $(C)$; diterpenes $(D)$; and fatty acids (E). Statistical difference among genotypes was tested using ANOVA followed by Tukey HSD $p<0.05$ for each treatment; letters indicate statistically significant groups.

See also Data S1. 
Undifferentiated cell

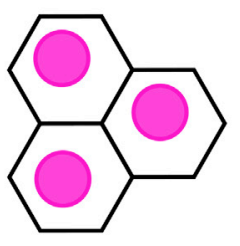

Apical notch

(proximal)

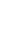

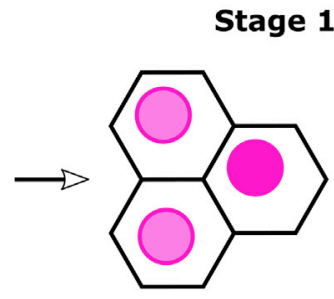

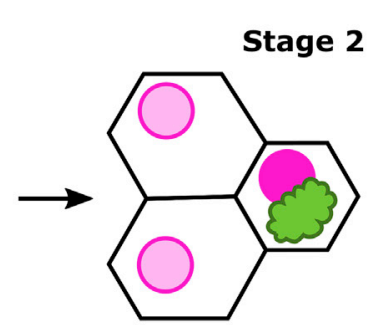

Figure 7. Proposed Model for MpC1HDZ Dynamics of Oil Body Cell Differentiation

All cells in proximity of an apical notch express MpC1HDZ. Although most of the cells gradually lose MpC1HDZ expression, some cells start expressing MpC1HDZ stronger than their neighbors (stage 1). These cells can form an oil body (stage 2). With distance from apical notch and maturation of oil body cell, MpC1HDZ expression fades (stage 3). During oil body cell development, neighboring cells continue expanding and dividing, although oil body cells remain smaller.

suggests that other transcriptional regulators could be relevant along with MpC1HDZ. For example, recently, MpERF13, a TF from the AP2-ERF family, was also shown to be involved in oil body cell differentiation [20].

We explored several environmental conditions where oil bodies could play a physiological role. Whereas we found no evidence that $\mathrm{Mpc} 1 \mathrm{hdz}$ plants were compromised in the response to an array of abiotic stressors, mutant plants were more susceptible to herbivory by the isopod $A$. vulgare (Figure $4 \mathrm{C}$ ) and mutant plant extracts were less efficient controlling the growth of B. subtilis (Figure 4D). Thus, our results provide genetic evidence supporting a role for oil bodies in liverwort defenses against arthropod herbivores, supporting the hypothesis formulated more than a century ago [27].

\section{Biochemical Properties of Mpc1hdz Mutants}

As expected, the paucity of oil bodies in Mpc1hdz plants resulted in a marked reduction in specific terpene-related compounds (Figure 6). It has been demonstrated that the sesquiterpenoids and bis(bibenzyls) are accumulated in the oil bodies [15]. These compounds present cytotoxic activity against bacteria and fungi and mammalian and plant cells [14, 15, 30-32] but also have other medical applications [60]. Indeed, such bioactivity is the likely reason $M$. polymorpha is mentioned at least as early as a Greek herbal in the first century as a surface poultice to prevent inflammation of wounds [68]. In planta, although we provide evidence of a role for oil bodies against isopods, additional functions in fungal or bacterial resistance have not been ruled out. However, that liverwort species with oil bodies in every cell form mycorrhizal fungal interactions [69] indicates fungi can avoid their cytotoxicity, perhaps due to oil bodies being membrane-bound organelles.

Oil bodies are thought to be a synapomorphy of this lineage that arose in the ancestral liverwort [7]. There is fossil evidence suggesting that selection pressure of herbivory was acting on ancestral liverworts [28, 70]. Thus, the anti-herbivory function of terpenoids and other secondary metabolites concentrated in oil bodies may date to the ancestral liverwort, as was also recently suggested in another study [71]. More broadly, as these compounds also play a central role in the ecology and evolution of the biotic response of seed plants [72], the origin and diversification of these secondary metabolites might have facilitated the evolution of early land plants by modulating their biotic interactions.

\section{Oil Body Cell Differentiation Depends on MpC1HDZ}

In $M$. polymorpha thalli, idioblasts emerge close to the apical notch in a proportion that later decreases due to unequal cell division rates [8]. Nonetheless, the precise mechanism of oil body differentiation and formation is still unclear. Here, we described Mpc1hdz mutants that not only have a severe reduction of oil bodies but also do not differentiate idioblast cells (Figure 1). Transcriptional analysis of Mpc1hdz thalli demonstrated that several downregulated genes encode proteins located in the oil body, such as TPS, MTPSL, MpABCG1, MpMYB02, and MpSYP12B [16, 62], together with other secondary metabolism-related genes that could be directly activated by MpC1HDZ (Figure 5). It is unclear whether the lethality encountered in MpC1HDZ gain-of-function mutants results from ectopic production of cytotoxic compounds, ectopic oil body cells, or another defect.

As oil bodies form at low frequency in Mpc1hdz mutants, other transcriptional regulators could be important for oil body cell differentiation. The R2R3-MYB TFs MpMYB02 and MpMYB14 control the bis(bibenzyl) and auronidin biosynthesis, respectively $[36,38]$. Mpc1hdz mutants showed a strong repression of MpMYB02 expression, whereas MpMYB14, important for the defense against the oomycete pathogen Phytophthora palmivora [73], remained unchanged. That $\mathrm{MpC} 1 \mathrm{HDZ}$ expression is not altered in MpMYBO2 gain-of-function alleles [36] suggests that MpMYBO2 is downstream MpC1HDZ activity; however, it is unknown whether MpMYB02 also expresses in oil body cells or participates in oil body cell differentiation and terpenoid biosynthesis. In contrast, we did not find any relationship of $M p C 1 H D Z$ with $M p M Y C X / Y$, which is associated with the defense-related induction of sesquiterpenoid biosynthesis in response to herbivory [71], or with ABA signaling, which is associated with bis(bibenzyl) increase under UV-C light stress [39].

Based on our observations, we propose a model for oil body cell differentiation (Figure 7). Undifferentiated cells derived from the notch express and accumulate MpC1HDZ to high levels: stage (1) a higher expression level of $\mathrm{MpC} 1 \mathrm{HDZ}$ in one 
of the cells promotes differentiation of an oil body cell, while $\mathrm{MpC} 1 \mathrm{HDZ}$ accumulation in neighboring cells decreases; stage (2) oil body forms in cells with high MpC1HDZ expression and oil body components are biosynthesized; and stage (3) oil bodies mature while $\mathrm{MpC} 1 \mathrm{HDZ}$ expression fades, and growth displaces them from the notch as non-idioblast cells continue dividing (Figure 7). We propose that MpC1HDZ mediates the developmental program of oil body cell differentiation. Notably, our results do not exclude that other transcription factors may act earlier than $\mathrm{MpC1HDZ}$ in oil body cell differentiation. Investigating how integration of different transcriptional and environmental inputs promotes oil body cell development and differentiation will help with further understanding of this process. Such information will also pave the way for manipulating oil body numbers and composition for biotechnological purposes.

\section{STAR $\star$ METHODS}

Detailed methods are provided in the online version of this paper and include the following:

- KEY RESOURCES TABLE

- RESOURCE AVAILABILITY

O Lead Contact

- Materials Availability

○ Data and Code Availability

- EXPERIMENTAL MODEL AND SUBJECT DETAILS

- METHOD DETAILS

O Marchantia transformation

O Genetic constructs

gRNA design and cloning

O MpC1HDZ cloning and genetic constructs

O Transcriptional reporters

O Translational reporters

Histology and microscopy

O GUS staining

O RT-qPCR analysis

Oil body cell and oil body distance measurement

O Oil body cell tracking

O Plant stress treatments

O Pill bug feeding assay

Ethanol extraction and overlay assay

RNA-seq

Hexane extraction and gas chromatography

- QUANTIFICATION AND STATISTICAL ANALYSIS

\section{SUPPLEMENTAL INFORMATION}

Supplemental Information can be found online at https://doi.org/10.1016/j. cub.2020.05.081.

\section{ACKNOWLEDGMENTS}

We thank Dr. Teruyuki Niimi (National Institute for Basic Biology) and Dr. Takahisa Miyatake (Okayama University) for identification of Armadillidium vulgare. The support of plant cultivation rooms was provided by the Model Plant Research Facility of National Institute for Basic Biology. We thank Dr. Magnus Eklund (Uppsala University) for his help with transcriptional reporters, Santiago Farías (UNL) for plant pictures, Dr. Feng Cheng (University of Tennessee) for helpful comments, Dr. Kimitsune Ishizaki (Kobe University) for the cloning vectors, and Dr. Keisuke Inoue for providing pMpGE_En04 and pBC-14. We thank
Dr. Saiko Yoshida and Dr. Peter Huijser for assistance with confocal imaging and image analysis, Michelle van der Gragt for GUS stainings of BoGa plants, and Dr. Hirofumi Nagakami for helpful discussions. This work was supported by Agencia I+D+i (PICT2017-1484 and PICT2018-00650 to J.E.M.) and CONICET (PIP267 to J.E.M.). F.R. is a doctoral fellow of CONICET. F.R. received a Travelling Fellowship from The Company of Biologists. J.E.M. is a CONICET career member. S.N.F. and J.L.B. were supported by the Australian Research Council (DP170100049). T.U. and T.K. were financially supported by Grantsin-Aid for Scientific Research from the Ministry of Education, Culture, Sports, Science and Technology of Japan (to T.U.: 17K19412, 18H02470, and19H05675; to T.K.: 17H07333 and 18K14738), The Mitsubishi Foundation, and Yamada Science Foundation. M.T. was supported by a core grant from the Max Planck Society and the Deutsche Forschungsgemeinschaft MAdLand SPP TS 229/4-1. S.Z. acknowledges support from the Deutsche Forschungsgemeinschaft (SFB 944).

\section{AUTHOR CONTRIBUTIONS}

F.R., E.B., J.L.B., M.T., and J.E.M. designed the research; M.T. and J.E.M. conceived the project; F.R., E.B., S.N.F., J.Q.D.G., R.A.M., T.D., S.Z., T.K., and T.U. performed research; and F.R., E.B., J.L.B., M.T., and J.E.M. wrote the paper.

\section{DECLARATION OF INTERESTS}

The authors declare no competing interests.

Received: March 10, 2020

Revised: May 3, 2020

Accepted: May 27, 2020

Published: June 18, 2020

\section{REFERENCES}

1. Delwiche, C.F., and Cooper, E.D. (2015). The evolutionary origin of a terrestrial flora. Curr. Biol. 25, R899-R910.

2. Bowman, J.L., Kohchi, T., Yamato, K.T., Jenkins, J., Shu, S., Ishizaki, K., Yamaoka, S., Nishihama, R., Nakamura, Y., Berger, F., et al. (2017). Insights into land plant evolution garnered from the Marchantia polymorpha genome. Cell 171, 287-304.e15.

3. Catarino, B., Hetherington, A.J., Emms, D.M., Kelly, S., and Dolan, L. (2016). The stepwise increase in the number of transcription factor families in the Precambrian predated the diversification of plants on land. Mol. Biol. Evol. 33, 2815-2819.

4. de Mendoza, A., Sebé-Pedrós, A., Šestak, M.S., Matejcic, M., Torruella, G., Domazet-Loso, T., and Ruiz-Trillo, I. (2013). Transcription factor evolution in eukaryotes and the assembly of the regulatory toolkit in multicellular lineages. Proc. Natl. Acad. Sci. USA 110, E4858-E4866.

5. Morris, J.L., Puttick, M.N., Clark, J.W., Edwards, D., Kenrick, P., Pressel, S., Wellman, C.H., Yang, Z., Schneider, H., and Donoghue, P.C.J. (2018) The timescale of early land plant evolution. Proc. Natl. Acad. Sci. USA 115, E2274-E2283.

6. Puttick, M.N., Morris, J.L., Williams, T.A., Cox, C.J., Edwards, D., Kenrick, P., Pressel, S., Wellman, C.H., Schneider, H., Pisani, D., et al. (2018). The interrelationships of land plants and the nature of the ancestral Embryophyte. Curr. Biol. 28, 733-745.e2.

7. Schuster, R.M. (1966). The Hepaticae and Anthocerotae of North AmericaVolume I (Columbia University).

8. Slavík, B. (1950). Specific bodies in the cells of hepatics (Hepaticae). Stud. Bot. Cech 11, 226-244.

9. Gottsche, C.M. (1843). Anatomisch-physiologische untersuchungen über Haplomitrium hookeri N.v.E., mit vergleichung anderer lebermoose. Nov. Actorum Acad. Caes. Leop.-Carol. Nat. Cur. 20, 267-398.

10. Lindberg, S.O. (1862). Ueber das vorkommen von aetherischen oelen in lebermoosen. Flora 45, 545-546. 
11. Lohmann, C.E.J. (1903). Beitrag zur chemie und biologie der lebermoose. Zentrallbl. 15, 215-256.

12. Müller, K. (1905). Beitrag zur kenntnis der ätherischen öle bei lebermoosen. Biol. Chem. 45, 299-319.

13. Asakawa, Y., and Ludwiczuk, A. (2018). Chemical constituents of bryophytes: structures and biological activity. J. Nat. Prod. 81, 641-660.

14. Chen, F., Ludwiczuk, A., Wei, G., Chen, X., Crandall-Stotler, B., and Bowman, J.L. (2018). Terpenoid secondary metabolites in bryophytes: chemical diversity, biosynthesis and biological functions. Crit. Rev. Plant Sci. 37, 210-231.

15. Tanaka, M., Esaki, T., Kenmoku, H., Koeduka, T., Kiyoyama, Y., Masujima, T., Asakawa, Y., and Matsui, K. (2016). Direct evidence of specific localization of sesquiterpenes and marchantin $A$ in oil body cells of Marchantia polymorpha L. Phytochemistry 130, 77-84.

16. Suire, C., Bouvier, F., Backhaus, R.A., Bégu, D., Bonneu, M., and Camara, B. (2000). Cellular localization of isoprenoid biosynthetic enzymes in Marchantia polymorpha. Uncovering a new role of oil bodies. Plant Physiol. 124, 971-978.

17. Pfeffer, W. (1874). Die oelkörper der lebermoose. Flora 57, 2-6, 17-27, 3343.

18. Pihakaski, K. (1968). A study of the ultrastructure of the shoot apex and leaf cells in two liverworts, with special reference to the oil bodies. Protoplasma 66, 79-103.

19. Suire, C. (2000). A comparative, transmission-electron microscopic study on the formation of oil-bodies in liverworts. J. Hattori Bot. Lab. 89, 209-232.

20. Kanazawa, T., Morinaka, H., Ebine, K., Shimada, T.L., Ishida, S., Minamino, N., Yamaguchi, K., Shigenobu, S., Kohchi, T., Nakano, A., et al. (2020). Switching secretory pathway direction for organelle acquisition in plants. bioRxiv. https://doi.org/10.1101/2020.03.02.956961.

21. Dombray, P. (1926). Contribution a l'étude des corpes oléiformes des hépatiques des environs de Nancy. PhD thesis (University of Paris).

22. Gavaudan, P. (1930/1). Recherches sur la cellule des hépatiques. Le Botaniste 22, 105-294.

23. Pressel, S., Duckett, J.G., Ligrone, R., and Proctor, M.C.F. (2009). Effects of de- and rehydration in desiccation-tolerant liverworts: a cytological and physiological study. Int. J. Plant Sci. 170, 182-199.

24. Hieronymous, G. (1892). Beiträge zur morphologie und biologie der algen. Beiträge zur Biologie der Pflanzen 5, 461-495.

25. Chalaud, G. (1931). Germination des spores et formation du game'tophyte chez Lophocolea cuspidata et Chiloscyphus polyanthus. Ann Bryol 4, 49-78.

26. Gavaudan, P. (1927). Sur l'origine et les caractéres des éléments oléiféres des Jungermaniales. C. R. Hebd. Seances Acad. Sci. 184, 1473-1475.

27. Stahl, E. (1888). Pflanzen und schnecken: biologische studie über die schutzmittel der pflanzen gegen schneckenfrass. Jena Z. Naturwiss. 22, 557-684.

28. Labandeira, C.C., Tremblay, S.L., Bartowski, K.E., and VanAller Hernick, L. (2014). Middle Devonian liverwort herbivory and antiherbivore defence. New Phytol. 202, 247-258.

29. Gahtori, D., and Chaturvedi, P. (2011). Antifungal and antibacterial potential of methanol and chloroform extracts of Marchantia polymorpha L. Arch. Phytopathol. Plant. Protect. 44, 726-731.

30. Asakawa, Y. (1990). Biologically active substances from bryophytes. In Bryophyte Development: Physiology and Biochemistry, R.N. Chopra, and S.C. Bhatla, eds. (CRC), pp. 259-287.

31. Banerjee, R.D., and Sen, S.P. (1979). Antibiotic activity of bryophytes. Bryologist 82, 141-153.

32. Komala, I., Ito, T., Yagi, Y., Nagashima, F., and Asakawa, Y. (2010). Volatile components of selected liverworts, and cytotoxic, radical scavenging and antimicrobial activities of their crude extracts. Nat. Prod. Commun. 5, 1375-1380.
33. Wu, Y.F., Zhao, Y., Liu, X.Y., Gao, S., Cheng, A.X., and Lou, H.X. (2018). A bHLH transcription factor regulates bisbibenzyl biosynthesis in the liverwort Plagiochasma appendiculatum. Plant Cell Physiol. 59, 1187-1199.

34. Kumar, S., Kempinski, C., Zhuang, X., Norris, A., Mafu, S., Zi, J., Bell, S.A., Nybo, S.E., Kinison, S.E., Jiang, Z., et al. (2016). Molecular diversity of terpene synthases in the liverwort Marchantia polymorpha. Plant Cell 28, 2632-2650.

35. Peters, K., Gorzolka, K., Bruelheide, H., and Neumann, S. (2018). Seasonal variation of secondary metabolites in nine different bryophytes. Ecol. Evol. 8, 9105-9117.

36. Kubo, H., Nozawa, S., Hiwatashi, T., Kondou, Y., Nakabayashi, R., Mori, T., Saito, K., Takanashi, K., Kohchi, T., and Ishizaki, K. (2018). Biosynthesis of riccionidins and marchantins is regulated by R2R3-MYB transcription factors in Marchantia polymorpha. J. Plant Res. 131, 849-864.

37. Arai, H., Yanagiura, K., Toyama, Y., and Morohashi, K. (2019). Genomewide analysis of MpBHLH12, a IIIf basic helix-loop-helix transcription factor of Marchantia polymorpha. J. Plant Res. 132, 197-209.

38. Albert, N.W., Thrimawithana, A.H., McGhie, T.K., Clayton, W.A., Deroles, S.C., Schwinn, K.E., Bowman, J.L., Jordan, B.R., and Davies, K.M (2018). Genetic analysis of the liverwort Marchantia polymorpha reveals that R2R3MYB activation of flavonoid production in response to abiotic stress is an ancient character in land plants. New Phytol. 218, 554-566.

39. Kageyama, A., Ishizaki, K., Kohchi, T., Matsuura, H., and Takahashi, K. (2015). Abscisic acid induces biosynthesis of bisbibenzyls and tolerance to UV-C in the liverwort Marchantia polymorpha. Phytochemistry 117, 547-553.

40. Romani, F., Reinheimer, R., Florent, S.N., Bowman, J.L., and Moreno, J.E. (2018). Evolutionary history of HOMEODOMAIN LEUCINE ZIPPER transcription factors during plant transition to land. New Phytol. 219, 408-421.

41. Romani, F., Ribone, P.A., Capella, M., Miguel, V.N., and Chan, R.L. (2016) A matter of quantity: Common features in the drought response of transgenic plants overexpressing HD-Zip | transcription factors. Plant Sci. 251, 139-154.

42. Cabello, J.V., Arce, A.L., and Chan, R.L. (2012). The homologous HD-Zip I transcription factors HaHB1 and AtHB13 confer cold tolerance via the induction of pathogenesis-related and glucanase proteins. Plant J. 69, 141-153.

43. González-Grandío, E., Pajoro, A., Franco-Zorrilla, J.M., Tarancón, C., Immink, R.G., and Cubas, P. (2017). Abscisic acid signaling is controlled by a BRANCHED1/HD-ZIP I cascade in Arabidopsis axillary buds. Proc. Natl. Acad. Sci. USA 114, E245-E254.

44. Ribone, P.A., Capella, M., and Chan, R.L. (2015). Functional characterization of the homeodomain leucine zipper I transcription factor AtHB13 reveals a crucial role in Arabidopsis development. J. Exp. Bot. 66, 59295943.

45. Moreno, J.E., Romani, F., and Chan, R.L. (2018). Arabidopsis thaliana homeodomain-leucine zipper type I transcription factors contribute to control leaf venation patterning. Plant Signal. Behav. 13, e1448334.

46. Sakakibara, K., Nishiyama, T., Sumikawa, N., Kofuji, R., Murata, T., and Hasebe, M. (2003). Involvement of auxin and a homeodomain-leucine zipper I gene in rhizoid development of the moss Physcomitrella patens. Development 130, 4835-4846.

47. Bowman, J.L., Araki, T., Arteaga-Vazquez, M.A., Berger, F., Dolan, L. Haseloff, J., Ishizaki, K., Kyozuka, J., Lin, S.S., Nagasaki, H., et al. (2016). The naming of names: guidelines for gene nomenclature in Marchantia. Plant Cell Physiol. 57, 257-261.

48. Tian, F., Yang, D.-C., Meng, Y.-Q., Jin, J., and Gao, G. (2020). PlantRegMap: charting functional regulatory maps in plants. Nucleic Acids Res. 48 (D1), D1104-D1113.

49. Zhao, Y., Ma, Q., Jin, X., Peng, X., Liu, J., Deng, L., Yan, H., Sheng, L., Jiang, H., and Cheng, B. (2014). A novel maize homeodomain-leucine zipper (HD-Zip) I gene, Zmhdz10, positively regulates drought and salt tolerance in both rice and Arabidopsis. Plant Cell Physiol. 55, 1142-1156. 
50. Gago, G.M., Almoguera, C., Jordano, J., Gonzalez, D.H., and Chan, R.L (2002). Hahb-4, a homeobox-leucine zipper gene potentially involved in abscisic acid-dependent responses to water stress in sunflower. Plant Cell Environ. 25, 633-640.

51. Cabello, J.V., Giacomelli, J.I., Gómez, M.C., and Chan, R.L. (2017). The sunflower transcription factor HaHB11 confers tolerance to water deficit and salinity to transgenic Arabidopsis and alfalfa plants. J. Biotechnol. 257, 35-46.

52. Ferreira, M.A., Pinheiro Da Cruz Waltenberg, F., Romano De Campos Pinto, E., and Grossi de Sa, M.F. (2013). Use of the coffee homeobox gene $<\mathrm{l}>\mathrm{CAHB} 12</ \mathrm{l}>$ to produce transgenic plants with greater tolerance to water scarcity and salt stress. International patent WO2012061911A9, filed November 12, 2010, and published May 18, 2012.

53. Bhattacharjee, A., Khurana, J.P., and Jain, M. (2016). Characterization of rice homeobox genes, OsHOX22 and OsHOX24, and over-expression of OsHOX24 in transgenic Arabidopsis suggest their role in abiotic stress response. Front. Plant Sci. 7, 627.

54. Olsson, A.S., Engström, P., and Söderman, E. (2004). The homeobox genes ATHB12 and ATHB7 encode potential regulators of growth in response to water deficit in Arabidopsis. Plant Mol. Biol. 55, 663-677.

55. Valdés, A.E., Overnäs, E., Johansson, H., Rada-Iglesias, A., and Engström, P. (2012). The homeodomain-leucine zipper (HD-Zip) class I transcription factors ATHB7 and ATHB12 modulate abscisic acid signalling by regulating protein phosphatase $2 \mathrm{C}$ and abscisic acid receptor gene activities. Plant Mol. Biol. 80, 405-418.

56. Ghosh, T.K., Kaneko, M., Akter, K., Murai, S., Komatsu, K., Ishizaki, K., Yamato, K.T., Kohchi, T., and Takezawa, D. (2016). Abscisic acid-induced gene expression in the liverwort Marchantia polymorpha is mediated by evolutionarily conserved promoter elements. Physiol. Plant. 156, 407-420.

57. Gehrmann, K. (1909). Zur befruchtungsphysiologie von Marchantia polymorpha L. Ber. Dtsch. Bot. Ges. 27, 341-348.

58. Goebel, K. (1898). Organographie der Pflanzen, Insbesondere der Archegoniaten und SamenpflanzenVolume 2, Third Edition (Gustav Fischer).

59. Proctor, M.C.F. (1990). The physiological basis of bryophyte production. Bot. J. Linn. Soc. 104, 61-77.

60. Asakawa, Y. (2008). Liverworts-potential source of medicinal compounds. Curr. Pharm. Des. 14, 3067-3088.

61. Jia, Q., Li, G., Köllner, T.G., Fu, J., Chen, X., Xiong, W., Crandall-Stotler, B.J., Bowman, J.L., Weston, D.J., Zhang, Y., et al. (2016). Microbialtype terpene synthase genes occur widely in nonseed land plants, but not in seed plants. Proc. Natl. Acad. Sci. USA 113, 12328-12333.

62. Kanazawa, T., Era, A., Minamino, N., Shikano, Y., Fujimoto, M., Uemura, T., Nishihama, R., Yamato, K.T., Ishizaki, K., Nishiyama, T., et al. (2016). SNARE molecules in Marchantia polymorpha: unique and conserved features of the membrane fusion machinery. Plant Cell Physiol. 57, 307-324.

63. Hofer, J., Turner, L., Moreau, C., Ambrose, M., Isaac, P., Butcher, S., Weller, J., Dupin, A., Dalmais, M., Le Signor, C., et al. (2009). Tendrilless regulates tendril formation in pea leaves. Plant Cell 21, 420-428.

64. Vlad, D., Kierzkowski, D., Rast, M.I., Vuolo, F., Dello Ioio, R., Galinha, C., Gan, X., Hajheidari, M., Hay, A., Smith, R.S., et al. (2014). Leaf shape evolution through duplication, regulatory diversification, and loss of a homeobox gene. Science 343, 780-783.

65. Perroud, P.F., Haas, F.B., Hiss, M., Ullrich, K.K., Alboresi, A., Amirebrahimi, M., Barry, K., Bassi, R., Bonhomme, S., Chen, H., et al. (2018). The Physcomitrella patens gene atlas project: large-scale RNAseq based expression data. Plant J. 95, 168-182.

66. Zwickel, W. (1932). Studien über die ocellen der lebermoose. Beihefte zum Botanischen Centralblatt 49, 569-648.

67. Garjeanne, A.J.M. (1903). Die ölkörper der Jungermanniales. Flora 92, 457-482.

68. Bowman, J.L. (2016). A brief history of Marchantia from Greece to genomics. Plant Cell Physiol. 57, 210-229.
69. Rimington, W.R., Pressel, S., Duckett, J.G., Field, K.J., Read, D.J., and Bidartondo, M.I. (2018). Ancient plants with ancient fungi: liverworts associate with early-diverging arbuscular mycorrhizal fungi. Proc. Biol. Sci. 285, 20181600.

70. Labandeira, C. (2007). The origin of herbivory on land: initial patterns of plant tissue consumption by arthropods. Insect Sci. 14, 259-275.

71. Peñuelas, M., Monte, I., Schweizer, F., Vallat, A., Reymond, P., GarcíaCasado, G., Franco-Zorrilla, J.M., and Solano, R. (2019). Jasmonaterelated MYC transcription factors are functionally conserved in Marchantia polymorpha. Plant Cell 31, 2491-2509.

72. Lange, B.M. (2015). The evolution of plant secretory structures and emergence of terpenoid chemical diversity. Annu. Rev. Plant Biol. 66, 139-159.

73. Carella, P., Gogleva, A., Hoey, D.J., Bridgen, A.J., Stolze, S.C., Nakagami, H., and Schornack, S. (2019). Conserved biochemical defenses underpin host responses to oomycete infection in an early-divergent land plant lineage. Curr. Biol. 29, 2282-2294.e5.

74. Flores-Sandoval, E., Eklund, D.M., and Bowman, J.L. (2015). A simple auxin transcriptional response system regulates multiple morphogenetic processes in the liverwort Marchantia polymorpha. PLoS Genet. 11, e1005207.

75. Busch, A., Deckena, M., Almeida-Trapp, M., Kopischke, S., Kock, C., Schüssler, E., Tsiantis, M., Mithöfer, A., and Zachgo, S. (2019). MpTCP1 controls cell proliferation and redox processes in Marchantia polymorpha. New Phytol. 224, 1627-1641.

76. Ishizaki, K., Nishihama, R., Ueda, M., Inoue, K., Ishida, S., Nishimura, Y., Shikanai, T., and Kohchi, T. (2015). Development of Gateway binary vector series with four different selection markers for the liverwort Marchantia polymorpha. PLoS ONE 10, e0138876.

77. Sugano, S.S., Nishihama, R., Shirakawa, M., Takagi, J., Matsuda, Y., Ishida, S., Shimada, T., Hara-Nishimura, I., Osakabe, K., and Kohchi, T. (2018). Efficient CRISPR/Cas9-based genome editing and its application to conditional genetic analysis in Marchantia polymorpha. PLoS ONE 13, e0205117.

78. Kim, D., Pertea, G., Trapnell, C., Pimentel, H., Kelley, R., and Salzberg, S.L. (2013). TopHat2: accurate alignment of transcriptomes in the presence of insertions, deletions and gene fusions. Genome Biol. 14, R36.

79. Robinson, J.T., Thorvaldsdóttir, H., Winckler, W., Guttman, M., Lander, E.S., Getz, G., and Mesirov, J.P. (2011). Integrative genomics viewer. Nat. Biotechnol. 29, 24-26.

80. Anders, S., Pyl, P.T., and Huber, W. (2015). HTSeq-a Python framework to work with high-throughput sequencing data. Bioinformatics 31, 166-169.

81. Afgan, E., Sloggett, C., Goonasekera, N., Makunin, I., Benson, D., Crowe, M., Gladman, S., Kowsar, Y., Pheasant, M., Horst, R., and Lonie, A. (2015). Genomics virtual laboratory: a practical bioinformatics workbench for the cloud. PLoS ONE 10, e0140829.

82. Adams, R.P. (2009). Identification of Essential Oil Components by Gas Chromatography/Mass Spectroscopy, Fourth Edition (Allured Pub.).

83. Ishizaki, K., Nishihama, R., Yamato, K.T., and Kohchi, T. (2016). Molecular genetic tools and techniques for Marchantia polymorpha research. Plant Cell Physiol. 57, 262-270.

84. Ishizaki, K., Chiyoda, S., Yamato, K.T., and Kohchi, T. (2008). Agrobacterium-mediated transformation of the haploid liverwort Marchantia polymorpha L., an emerging model for plant biology. Plant Cell Physiol. 49, 1084-1091.

85. Kubota, A., Ishizaki, K., Hosaka, M., and Kohchi, T. (2013). Efficient Agrobacterium-mediated transformation of the liverwort Marchantia polymorpha using regenerating thalli. Biosci. Biotechnol. Biochem. 77, 167-172.

86. Sugano, S.S., Shirakawa, M., Takagi, J., Matsuda, Y., Shimada, T., HaraNishimura, I., and Kohchi, T. (2014). CRISPR/Cas9-mediated targeted mutagenesis in the liverwort Marchantia polymorpha L. Plant Cell Physiol. 55, 475-481.

87. Hisanaga, T., Okahashi, K., Yamaoka, S., Kajiwara, T., Nishihama, R., Shimamura, M., Yamato, K.T., Bowman, J.L., Kohchi, T., and Nakajima, 
K. (2019). A cis-acting bidirectional transcription switch controls sexual dimorphism in the liverwort. EMBO J. 38, e100240.

88. Eshed, Y., Baum, S.F., and Bowman, J.L. (1999). Distinct mechanisms promote polarity establishment in carpels of Arabidopsis. Cell 99, 199-209.

89. Barbier de Reuille, P., Routier-Kierzkowska, A.L., Kierzkowski, D., Bassel, G.W., Schüpbach, T., Tauriello, G., Bajpai, N., Strauss, S., Weber, A., Kiss, A., et al. (2015). MorphoGraphX: a platform for quantifying morphogenesis in 4D. elife 4, 05864.

90. Ré, D.A., Capella, M., Bonaventure, G., and Chan, R.L. (2014). Arabidopsis AtHB7 and AtHB12 evolved divergently to fine tune processes associated with growth and responses to water stress. BMC Plant Biol. 14, 150.

91. Nakazaki, A., Yamada, K., Kunieda, T., Sugiyama, R., Hirai, M.Y., Tamura, K., Hara-Nishimura, I., and Shimada, T. (2019). Leaf endoplasmic reticulum bodies identified in Arabidopsis rosette leaves are involved in defense against herbivory. Plant Physiol. 179, 1515-1524.
92. Hockett, K.L., and Baltrus, D.A. (2017). Use of the soft-agar overlay technique to screen for bacterially produced inhibitory compounds. J. Vis. Exp. $119,55064$.

93. Flores-Sandoval, E., Romani, F., and Bowman, J.L. (2018). Co-expression and transcriptome analysis of Marchantia polymorpha transcription factors supports class $\mathrm{C}$ ARFs as independent actors of an ancient auxin regulatory module. Front. Plant Sci. 9, 1345.

94. Montgomery, S.A., Tanizawa, Y., Galik, B., Wang, N., Ito, T., Mochizuki, T. Akimcheva, S., Bowman, J.L., Cognat, V., Maréchal-Drouard, L., et al (2020). Chromatin organization in early land plants reveals an ancestral association between H3K27me3, transposons, and constitutive heterochromatin. Curr. Biol. 30, 573-588.e7.

95. Davies, K.M., Jibran, R., Zhou, Y., Albert, N.W., Brummell, D.A., Jordan, B.R., Bowman, J.L., and Schwinn, K.E. (2020). The evolution of flavonoid biosynthesis: a bryophyte perspective. Front. Plant Sci. 11, 7. 
Current Biology

Article

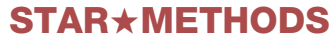

KEY RESOURCES TABLE

\begin{tabular}{|c|c|c|}
\hline REAGENT or RESOURCE & SOURCE & IDENTIFIER \\
\hline \multicolumn{3}{|l|}{ Bacterial Strains } \\
\hline $\begin{array}{l}\text { E. coli DH } 5 \alpha \text { chemically } \\
\text { competent cells }\end{array}$ & Our lab collection & $\mathrm{N} / \mathrm{A}$ \\
\hline $\begin{array}{l}\text { E. coli } \mathrm{DH} 10 \mathrm{~B} \text { chemically } \\
\text { competent cells }\end{array}$ & Our lab collection & N/A \\
\hline $\begin{array}{l}\text { Agrobacterium tumefaciens } \\
\text { GV3001 }\end{array}$ & Our lab collection & $\mathrm{N} / \mathrm{A}$ \\
\hline $\begin{array}{l}\text { Agrobacterium tumefaciens } \\
\text { GV3101 }\end{array}$ & Our lab collection & $\mathrm{N} / \mathrm{A}$ \\
\hline $\begin{array}{l}\text { Agrobacterium tumefaciens } \\
\text { LBA4404 }\end{array}$ & Our lab collection & $\mathrm{N} / \mathrm{A}$ \\
\hline Bacillus subtilis & Our lab collection & $\mathrm{N} / \mathrm{A}$ \\
\hline \multicolumn{3}{|c|}{ Chemicals, Peptides, and Recombinant Proteins } \\
\hline $\begin{array}{l}\text { NEBuilder HiFi DNA } \\
\text { Assembly Master Mix }\end{array}$ & New England BioLabs & Cat\# E2621 \\
\hline $\begin{array}{l}\text { Gateway LR clonase II } \\
\text { enzyme mix }\end{array}$ & ThermoFisher & Cat\# 11791020 \\
\hline $\begin{array}{l}\text { MMLV reverse transcriptase } \\
\|\end{array}$ & Promega & Cat\# M1701 \\
\hline Taq Pegasus Polymerase & PBL & Cat\# EA0101 \\
\hline $\begin{array}{l}\text { PrimeSTAR GXL DNA } \\
\text { Polymerase }\end{array}$ & Takara Bio & Cat\# R050A \\
\hline Roche SYBR mix & Roche Life Science & Cat\# 4887352001 \\
\hline Cefotaxime sodium salt & Sigma-Aldrich & Cat\# C7039 \\
\hline Hygromycin B & Melford & Cat\# H7502 \\
\hline Chlorsulfuron & Sigma-Aldrich & Cat\# 34322 \\
\hline G418 Melford Cat\#G0175 & Melford & Cat\# G0175 \\
\hline Carbenicillin & Sigma-Aldrich & Cat\# C3416 \\
\hline Propidium iodide & Sigma-Aldrich & Cat\# P4170 \\
\hline Plant agar & Phytotechlab & Cat\# A175 \\
\hline Gamborg B-5 basal médium & Phytotechlab & Cat\# G398 \\
\hline $\begin{array}{l}\text { Gamborg B5 medium } \\
\text { including vitamins }\end{array}$ & Duchefa Biochemie & Cat\# G0210 \\
\hline Agar-Agar, Kobe I & Carl Roth & Cat\# 5210 \\
\hline Tween 20 & Carl Roth & Cat\# 9127 \\
\hline Nile Red & Sigma-Aldrich & Cat\# 72485 \\
\hline $\begin{array}{l}\text { BODIPY } 493 / 503 \text { (4,4- } \\
\text { Difluoro-1,3,5,7,8- } \\
\text { Pentamethyl-4-Bora-3a,4a- } \\
\text { Diaza-s-Indacene) }\end{array}$ & Thermo Fisher Scientific & Cat\# D3922 \\
\hline $\begin{array}{l}\text { Potassium } \\
\text { hexacyanoferrate(II) } \\
\text { trihydrate }\end{array}$ & Carl Roth & Cat\# P745 \\
\hline $\begin{array}{l}\text { Potassium } \\
\text { hexacyanoferrate(III) }\end{array}$ & Merck & Cat\# 4973 \\
\hline $\mathrm{X}$-GlcA & Melford & Cat\# MB1021 \\
\hline Hexane & Merck & Cat\# 104369 \\
\hline & & (Continued c \\
\hline
\end{tabular}




\begin{tabular}{|c|c|c|}
\hline \multicolumn{3}{|l|}{ Continued } \\
\hline REAGENT or RESOURCE & SOURCE & IDENTIFIER \\
\hline $\begin{array}{l}\text { Tris-(perfluorobutyl)-amine } \\
\text { (FC-43) }\end{array}$ & Agilent & Cat\# 392035300 \\
\hline Helium & BOC Group & Cat\# 220 \\
\hline Limonene & Sigma-Aldrich & Cat\# 183164 \\
\hline Aromadendrene & Sigma-Aldrich & Cat\# 11067 \\
\hline Valencene & Sigma-Aldrich & Cat\# W344303 \\
\hline Caryophyllene oxide & Sigma-Aldrich & Cat\# 22076 \\
\hline Hexadecane & Sigma-Aldrich & Cat\# 52209 \\
\hline Palmitic acid & Sigma-Aldrich & Cat\# P0500 \\
\hline Phytol & Sigma-Aldrich & Cat\# W502200 \\
\hline Stigmasterol & Sigma-Aldrich & Cat\# S2424 \\
\hline F.A.M.E. Mix C8-C24 & Supelco & Cat\# CRM18918 \\
\hline \multicolumn{3}{|l|}{ Critical Commercial Assays } \\
\hline $\begin{array}{l}\text { QIAprep spin miniprep kit } \\
\text { QIAGEN Cat\#27106 }\end{array}$ & QIAGEN & Cat\# 27106 \\
\hline NucleoSpin Plasmid (NoLid) & Macherey-Nagel & Cat\# 740499 \\
\hline Turbo DNA-free kit & Invitrogen & Cat\# AM1907 \\
\hline $\begin{array}{l}\text { TruSeq RNA Library Prep Kit } \\
\text { v2 }\end{array}$ & Illumina & Cat\# RS-122-2001 \\
\hline RNeasy Plant Kit & QIAGEN & Cat\# 74904 \\
\hline \multicolumn{3}{|l|}{ Deposited Data } \\
\hline RNA-seq data & This study & Bioproject \# PRJNA615642 \\
\hline RNA-seq analysis & This study; Mendeley Data & $\begin{array}{l}\text { https://doi.org/10.17632/ } \\
\text { 7xf7gktjjg.2 }\end{array}$ \\
\hline $\begin{array}{l}\text { Marchantia polymorpha } \\
\text { reference genome v3.1 }\end{array}$ & [2] & http://marchantia.info/ \\
\hline \multicolumn{3}{|c|}{ Experimental Models: Organisms/Strains } \\
\hline $\begin{array}{l}\text { Marchantia polymorpha } \\
\text { subsp. ruderalis Australian/ } \\
\text { Melbourne accession male } \\
\text { (WT male) }\end{array}$ & $\begin{array}{l}\text { Dr. John Bowman, Monash } \\
\text { University [74] }\end{array}$ & N/A \\
\hline $\begin{array}{l}\text { Marchantia polymorpha } \\
\text { subsp. ruderalis Australian/ } \\
\text { Melbourne accession female } \\
\text { (WT female) }\end{array}$ & $\begin{array}{l}\text { Dr. John Bowman, Monash } \\
\text { University [74] }\end{array}$ & N/A \\
\hline $\begin{array}{l}\text { Marchantia polymorpha } \\
\text { subsp. ruderalis Osnabrück } \\
\text { Botanical Garden (BoGa) }\end{array}$ & $\begin{array}{l}\text { Dr. Sabine Zachgo, } \\
\text { University of Osnabrück [75] }\end{array}$ & N/A \\
\hline Nicotiana benthamiana & Our lab collection & N/A \\
\hline Armadillidium vulgare & Myodaiji area (Aichi, Japan) & N/A \\
\hline $\begin{array}{l}\text { WT Mpc1hdz-1 }{ }^{\text {ge }} \text { o }(\Delta 32 b p \\
\text { frameshift) }\end{array}$ & $\begin{array}{l}\text { This study, nickase Cas9, } \\
\text { sgRNA-2 (Figure } 1 \text { A) }\end{array}$ & N/A \\
\hline $\begin{array}{l}\text { WT Mpc1hdz-2ge } q(\Delta 1 b p \\
\text { frameshift) }\end{array}$ & $\begin{array}{l}\text { This study, nickase Cas9, } \\
\text { sgRNA-2 (Figure } 1 \mathrm{~A} \text { ) }\end{array}$ & N/A \\
\hline $\begin{array}{l}\text { BoGa Mpc } 1 h d z-3^{g e} q \\
\text { ( } \triangle \text { whole gene) }\end{array}$ & $\begin{array}{l}\text { This study, conventional } \\
\text { Cas9 using sgRNA-4 and } \\
\text { sgRNA-5 (Figure 1A) }\end{array}$ & N/A \\
\hline BoGa Mpc1hdz-4 $4^{g e} \delta^{\hat{*}}(\Delta H D)$ & $\begin{array}{l}\text { This study, conventional } \\
\text { Cas9 using sgRNA-1 and } \\
\text { sgRNA-3 (Figure 1A) }\end{array}$ & N/A \\
\hline pro $E F 1: M p C 1 H D Z_{m u t}$ & This study & N/A \\
\hline $\begin{array}{l}\text { pro35S:MpC1HDZ } \text { mut }^{-} \\
\text {CITRINE }\end{array}$ & This study & $\mathrm{N} / \mathrm{A}$ \\
\hline
\end{tabular}


Current Biology

\begin{tabular}{|c|c|c|}
\hline \multicolumn{3}{|l|}{ Continued } \\
\hline REAGENT or RESOURCE & SOURCE & IDENTIFIER \\
\hline $\begin{array}{l}\text { BoGa Mpc1hdz-3ge } \\
\text { proMpC1HDZ }{ }^{3 k b}: \mathrm{MpC} 1 \mathrm{HDZ}- \\
\text { Citrine }\end{array}$ & This study & N/A \\
\hline $\begin{array}{l}\text { BoGa Mpc1hdz-3 } \\
\text { proMpC1HDZ } \\
\text { tdTomato }\end{array}$ & This study & N/A \\
\hline 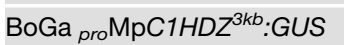 & This study & N/A \\
\hline 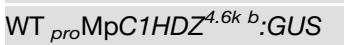 & This study & $\mathrm{N} / \mathrm{A}$ \\
\hline $\begin{array}{l}\text { proMpC1HDZ } Z^{4.6 k b}- \\
M p C 1 H D Z_{\text {mut }} \text {-Citrine }\end{array}$ & This study & N/A \\
\hline pro35S:GFP-MpC1HDZ & This study & $\mathrm{N} / \mathrm{A}$ \\
\hline \multicolumn{3}{|l|}{ Oligonucleotides } \\
\hline $\begin{array}{l}\text { A list of all oligonucleotides } \\
\text { used in this study can be } \\
\text { found in Table S3 }\end{array}$ & This study & $\mathrm{N} / \mathrm{A}$ \\
\hline \multicolumn{3}{|l|}{ Recombinant DNA } \\
\hline pSKF-HART & [74] & $\mathrm{N} / \mathrm{A}$ \\
\hline pEF1proBJ36v2.0 & [74] & $\mathrm{N} / \mathrm{A}$ \\
\hline pMpGWB104 & [76] & Addgene Cat\# 68558 \\
\hline pMpGWB307 & [76] & Addgene Cat\# 68635 \\
\hline pMpGWB329 & [76] & Addgene Cat\# 68657 \\
\hline pMpGWB403 & [76] & Addgene Cat\# 68668 \\
\hline pMpGWB406 & [76] & Addgene Cat\# 68644 \\
\hline pGWB407 & [76] & Addgene Cat\# 74801 \\
\hline pMpGE_En03 & [77] & Addgene Cat\# 71535 \\
\hline pMpGE_En04 & modified from pMpGE_En03 & N/A \\
\hline pMpGE010 & {$[77]$} & Addgene Cat\# 71536 \\
\hline $\begin{array}{l}\text { pMpGE_En03- } \\
\text { MpC1HDZ_gRNA1 }\end{array}$ & This study & N/A \\
\hline pMpGE_En04-gRNA-wg-1 & This study & N/A \\
\hline pMpGE_En04-gRNA-wg-2 & This study & $\mathrm{N} / \mathrm{A}$ \\
\hline pMpGE_En04-gRNA-HD-1 & This study & $\mathrm{N} / \mathrm{A}$ \\
\hline pMpGE_En04-gRNA-HD-2 & This study & $\mathrm{N} / \mathrm{A}$ \\
\hline${ }_{\text {pro }} E F 1: M p C 1 H D Z_{m u t}$ & This study & $\mathrm{N} / \mathrm{A}$ \\
\hline pro35S:MpC1HDZ $Z_{\text {mut }}$-Citrine & This study & $\mathrm{N} / \mathrm{A}$ \\
\hline $\begin{array}{l}\text { proMpC1HDZ }{ }^{3 k b}: \mathrm{MpC} 1 \mathrm{HDZ}- \\
\text { tdTomato }\end{array}$ & This study & $\mathrm{N} / \mathrm{A}$ \\
\hline pro $M p C 1 H D Z^{3 k b}: G U S$ & This study & $\mathrm{N} / \mathrm{A}$ \\
\hline pro MpC1HDZ ${ }^{4.6 k b}: G U S$ & This study & $\mathrm{N} / \mathrm{A}$ \\
\hline 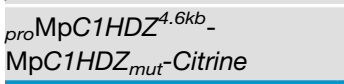 & This study & $\mathrm{N} / \mathrm{A}$ \\
\hline \multicolumn{3}{|l|}{ Software and Algorithms } \\
\hline ImageJ (Fiji) & $\begin{array}{l}\text { https://imagej.net/Fiji/ } \\
\text { Downloads }\end{array}$ & V2.0.0-rc-62/1.51s \\
\hline Rstudio & https://www.rstudio.com/ & V1.1.383 \\
\hline Primer-blast & $\begin{array}{l}\text { https://www.ncbi.nlm.nih. } \\
\text { gov/tools/primer-blast/ }\end{array}$ & N/A \\
\hline Photoshop software & Adobe systems, USA & $\mathrm{N} / \mathrm{A}$ \\
\hline TopHat v2.1.0 for Galaxy & [78] & $\mathrm{N} / \mathrm{A}$ \\
\hline $\begin{array}{l}\text { Integrative genomics viewer } \\
\text { IGV }\end{array}$ & [79] & V1.3.1 \\
\hline HTseq-code using Galaxy & [80] & N/A \\
\hline
\end{tabular}




\begin{tabular}{lll}
\hline Continued & & \\
\hline REAGENT or RESOURCE & SOURCE & IDENTIFIER \\
\hline EdgeR-package for Galaxy & {$[81]$} & N/A \\
R software & N/A & V3.6.0 \\
$\begin{array}{l}\text { Sesquiterpenes mass } \\
\text { spectra library }\end{array}$ & {$[82]$} & N/A \\
Agilent MSD ChemStation & Agilent & E.02.02.1431 \\
NIST 11 database & https://chemdata.nist.gov/ & N/A \\
& $\begin{array}{l}\text { dokuwiki/doku.php? } \\
\text { id=chemdata:start }\end{array}$ & \\
\hline Other & & This study; Mendeley Data \\
\hline Sequence data analyses & & https://doi.org/10.17632/ \\
\hline
\end{tabular}

\section{RESOURCE AVAILABILITY}

\section{Lead Contact}

Further information and requests for resources and reagents should be directed to and will be fulfilled by the Lead Contact, Javier E. Moreno (javier.moreno@santafe-conicet.gov.ar).

\section{Materials Availability}

No new reagents were generated in this study.

Data and Code Availability

The datasets generated during this study have been deposited in a public repository under the accession Bioproject \# PRJNA615642. Analyzed RNA-seq data for Figure 5 in the paper is available at Mendeley Data: https://doi.org/10.17632/ 7xf7gktjjg.2.

\section{EXPERIMENTAL MODEL AND SUBJECT DETAILS}

Marchantia polymorpha sp. ruderalis Australian (Melbourne, AU) ecotype [74] and BoGA ecotype (obtained from Botanical Garden in Osnabrück, Germany) [75] were cultivated from gemmae under axenic conditions on vented 9 by $2.5 \mathrm{~cm}$ Petri dishes containing $1 / 2$ strength Gamborg's B5 medium pH 5.5 solidified with 1\% (w/v) agar, referred as normal media, under long days conditions (80$100 \mu \mathrm{mol} \mathrm{m}{ }^{-2} \mathrm{~s}^{-1}$ ) at $22^{\circ} \mathrm{C}$. Experimental treatments are described below. BoGa ecotype was grown in growth chambers with continuous white light at $50 \mu \mathrm{mol} \mathrm{m} \mathrm{s}^{-1}$ at $22^{\circ} \mathrm{C}$, unless stated otherwise. Sexual reproduction was induced using white light supplemented with far-red light (730 nm, Cree LEDs) as described before [83]. Plants were crossed in sterile conditions using autoclaved water for spermatozoid transfer. Crossing was repeated twice and plants were returned to long day condition until sporangia were ready for harvest. Sporangia were dried and stored at $-80^{\circ} \mathrm{C}$.

\section{METHOD DETAILS}

\section{Marchantia transformation}

M. polymorpha spore transformation was performed as described previously using both ecotypes [84]. Briefly, M. polymorpha spores were germinated and grown in $25 \mathrm{~mL} 1 / 2$ strength liquid Gamborg's B5 with $2 \%(w / v)$ sucrose, 0.1\%(w/v) Casamino acids and $0.03 \%(\mathrm{w} / \mathrm{v})$ L-Glutamine for 10 days prior to co-cultivation with $1 \mathrm{~mL}$ of an Agrobacterium tumefaciens (GV3001) suspension, harboring binary plasmids, along with acetosyringone to a final concentration of $100 \mu \mathrm{M}$. Sporelings were plated on selection media (10 $\mu \mathrm{g} / \mathrm{mL}$ Hygromycin B with $200 \mathrm{mg} / \mathrm{mL}$ Timentin). After two weeks, T1 lines were transferred for one more week to new selection plates before being transferred to standard growth plates for gemmae production. Gemmae (G1 or G2 generation) from several independent primary transformants ( $\mathrm{T} 1$ generation) were analyzed for the presence of the transgene by PCR and sequencing, with some selected for further analysis.

Translational reporters were transformed into $\mathrm{Mpc} 1 \mathrm{hdz}-3^{g e}$ mutant using Agrobacterium-mediated transformation of regenerating thalli [85]. Briefly, plants were grown from gemmae on standard medium in continuous light for 14 days. 14 day-old thalli were cut into four pieces without apical notch and cultured for 3 days on standard medium with $1 \%$ sucrose to induce regeneration. Regenerating thalli were co-cultured with $A$. tumefaciens GV3101 in liquid medium with $100 \mu \mathrm{M}$ acetosyringone with agitation. After three days the plants were washed six times with autoclaved water, incubated for $30 \mathrm{~min}$ in $1 \mathrm{~g} / \mathrm{L}$ cefotaxime and plated on selective plates 
containing $0.6 \mu \mathrm{M}$ chlorsulfuron and $120 \mu \mathrm{g} / \mathrm{mL}$ cefotaxime. After transformants appeared, they were transferred to new selective plates. G2 generation was analyzed for fluorescence and presence of the transgene by PCR.

\section{Genetic constructs}

All primer sequences are available in Table S3.

\section{gRNA design and cloning}

Synthetic guide RNAs (gRNA) targeting genes of interest were co-expressed on independent T-DNAs using pGE010 plasmid [77, 86]. Design of specific gRNAs was performed such that putative off-targets ( $M$. polymorpha genome assembly v3.1) lacked an associated protospacer adjacent motif (PAM) site. For the generation of mutant alleles 1 and 2 we used single gRNAs transformation, primers MpC1HDZ_gRNA1 were cloned downstream of the MpU6 promoter, excluding PAM sites, in pMpGE_En03 using Bsal sites and were co-expressed with Cas9 [86]. For the double gRNAs transformations to generate mutant alleles 3 and 4, gRNAs were designed using the online tool http://marchantia.info/casfinder. The first gRNAs (gRNA-wg-1 or gRNA-HD-1) were cloned into pMpGE_En04 using Bsal sites [87]. Second gRNAs (gRNA-wg-2 or gRNA-HD-2) were cloned into pMpBC_GE14 using Bsal sites. Both vectors were digested using Bgll. The pMpGE_En04 backbone with the first gRNA and the fragment with the second gRNA were ligated, resulting in pMpGE_En04 with both gRNAs in tandem each under the control of the MpU6 promoter. These vectors were used in a Gateway LR reaction with the binary vector pMpGE010 featuring Arabidopsis-codon-optimized Cas9 nuclease under the control of the EF1 promoter $[77,86,87]$.

\section{MpC1HDZ cloning and genetic constructs}

The MpC1HDZ (Mp3g02320) coding sequence from M. polymorpha Tak-1 genome v3.1 (http://www. marchantia.info) was used to design specific primers. The cDNA was subcloned into pENTR-DTOPO (Thermo Fisher, Waltham, MA, US; pENTR-MpC1HDZ) using primers $\mathrm{MpC} 1 \mathrm{HDZ} \_\mathrm{CACC} \_\mathrm{fw}$ and $\mathrm{MpC} 1 \mathrm{HDZ} \_r v$. The CDS was mutagenized using primers MpC1HDZ_gibson_fw and MpC1HDZ_mut_rv, and MpC1HDZ_mut_fw and MpC1HDZ_gibson_rv in order to make it resistant to the gRNA. Fragments were purified and used as template for a subsequent amplification using primers MpC1HDZ_gibson_fw and MpC1HDZ_gibson_rv and cloned in a pENTR2B (Thermo Fisher, Waltham, MA, US) using NEBuilder HiFi DNA Assembly Master Mix (New England BioLabs, UK, pE2B-MpC1HDZ mut ). Later, it was subsequently recombined using the Gateway LR Clonase II Enzyme Mix (Thermo Fisher, Waltham, MA, US) according to the manufacturer's instructions into different destination vectors: pMpGWB403 (pro EF1:MpC1HDZ ${ }_{\text {mut }}$ ), pMpGWB406 (pro35S:MpC1HDZmut-Citrine) [76]. As a control, a $4.6 \mathrm{~kb}$ promoter region of MpC1HDZ was amplified with primers proMpC1HDZ_gibson_fw and proMpC1HDZ_gibson_fw as described below. Both fragments were used for cloning in a pENTR2B with NEBuilder HiFi DNA Assembly Master Mix and recombined in pGWB407 (pro MpC1HDZ ${ }^{4.6 k b}-\mathrm{MpC1HDZ}_{\text {mut }}{ }^{-}$Citrine).

\section{Transcriptional reporters}

The promoter sequence of $\mathrm{MpC1HDZ}$ was obtained from Phytozome. A $4.6 \mathrm{~kb}$ fragment of the promoter was amplified using primers proMpC1HDZ-Ndel-fw and proMpC1HDZ-Sall-rv and cloned into pCRII-TOPO. It was subsequently subcloned into pRITA, which has a multiple cloning site upstream of a GUS/UidA reporter gene and nos terminator [88], using Ndel and Kpnl. Finally, pro MpC1HDZ ${ }^{4.6 k b}:$ GUS was cloned into pSKF-KART using Notl [74].

Alternatively, a $3 \mathrm{~kb}$ sequence upstream of $\mathrm{MpC} 1 \mathrm{HDZ}$ start was amplified from BoGa DNA using GXL polymerase and primers with attB1 and attB2 sites. The subsequent PCR product was recombined with pDONR201 in BP reaction. The entry clone was recombined in an LR reaction with pMpGWB104 to obtain GUS reporter [76].

\section{Translational reporters}

To obtain translational reporters, cDNA was obtained from total RNA extracted from BoGa ecotype thalli. MpC1HDZ was amplified from cDNA using a specific forward primer and polyT reverse primer (Roche) and ligated into pGEMT. The amplified CDS starts at position 3 and ends at position 720 relative to the genome prediction and therefore likely represents a shorter transcript. MpC1HDZ CDS was amplified again from this plasmid to add Spel sites. The $3 \mathrm{~kb}$ sequence upstream of MpC1HDZ CDS start was amplified from BoGa DNA and ligated into pGEMT vector. MpC1HDZ CDS was inserted behind the $3 \mathrm{~kb}$ MpC1HDZ promoter using Spel restriction sites. The $3 \mathrm{~kb} \mathrm{MpC} 1 \mathrm{HDZ}$ promoter and MpC1HDZ CDS combination were amplified using primers with attB1 and attB2 sites and recombined in BP reaction with pDONR201. The entry clone was recombined in LR reaction with pMpGWB307 destination vector to obtain citrine fusion, or with pMpGWB329 to obtain tdTomato fusion [76].

\section{Histology and microscopy}

Fresh M. polymorpha gemmae were resuspended in $0.1 \%(\mathrm{v} / \mathrm{v})$ Triton X-100 and observed using a compound microscope (Nikon Eclipse E200, Nikon Instruments, Melville, NY, USA; or Zeiss Axio Imager M2) or Leica MZ10F stereomicroscope (Leica Microsystems, Wetzlar, Germany; or Nikon SMZ1500). In the case of thallus and cup cross sections, fresh thalli were sliced into 0.5-0.8 mm thick slices manually using a razor blade and observed in the compound microscope.

Oil body staining was performed using a $10 \mathrm{mg} \mathrm{mL}^{-1}$ Nile Red dissolved in phosphate buffered saline (PBS) just before use as described before [15]. This solution was vacuum infiltrated for $1 \mathrm{~min}$, incubated $10 \mathrm{~min}$ at room temperature and then rinsed with 


\section{CellPress}

\section{Current Biology}

$0.1 \%(\mathrm{w} / \mathrm{v})$ Triton X-100 solution. For BODIPY staining, the stock solution was diluted 1000 times in PBS to prepare the working solution. Plants were covered with BODIPY for $10 \mathrm{~min}$ in darkness and washed three times with PBS.

The samples were observed using GFP filter under fluorescent microscope (Zeiss Axio Imager M2). Oil bodies in gemmae were manually counted from images. Thallus area was quantified at different time intervals from pictures with ImageJ software. Thallus growth rate is the proportion between final and initial area of the thallus. All data was analyzed and plotted in R with built-in packages.

\section{GUS staining}

GUS assays were performed as described previously [74]. Plants were immersed in GUS staining solution (100 mM Potassium Ferrocyanide, $100 \mathrm{mM}$ Potassium Ferricyanide and $1 \mathrm{mM} \mathrm{X-Gluc)}$ and after applying 2 rounds of vacuum for 1 min, the plants were incubated for $1.5 \mathrm{~h}$ to $2 \mathrm{~h}$ at $37^{\circ} \mathrm{C}$ in darkness, cleared with ethanol, and imaged using a compound microscope or stereoscopic microscope. BoGa ecotypes were stained similarly but with slightly different GUS staining solution composition (50 mM phosphate buffer, $10 \mathrm{mM}$ EDTA, $50 \mathrm{mM}$ Potassium Ferrocyanide, $50 \mathrm{mM}$ Potassium Ferricyanide and $1.4 \mathrm{mM} \mathrm{X-Gluc)}$ and the stain was vacuum-infiltrated for $10 \mathrm{~min}$ before incubation at $37^{\circ} \mathrm{C}$.

\section{RT-qPCR analysis}

Total RNA was purified from 3-week-old M. polymorpha plants using Trizol reagent (Invitrogen, Carlsbad, CA, USA) according to the manufacturer's instructions. $1 \mu \mathrm{g}$ of RNA was reverse-transcribed using oligo(dT)18 and MMLV reverse transcriptase II (Promega, Madison, WA, USA). Quantitative real-time PCR (qPCR) was performed using a StepOnePlus Real-Time PCR System (Applied Biosystems); each reaction contained a final volume of $20 \mu \mathrm{L}$ that included $2 \mu \mathrm{L}$ of SYBR green ( $4 \mathrm{x}), 8 \mathrm{pmol}$ of each primer, $2 \mathrm{mM} \mathrm{MgCl} 2$, $10 \mu \mathrm{L}$ of a $1 / 15$ dilution of the RT reaction and $0.1 \mu \mathrm{L}$ of Taq Polymerase (Invitrogen). Thermocycler parameters were as follows: $94^{\circ} \mathrm{C}$ $12 \mathrm{~s}, 60^{\circ} \mathrm{C} 12 \mathrm{~s}, 72^{\circ} \mathrm{C} 12 \mathrm{~s}$. Fluorescence was quantified over 40 cycles at $72^{\circ} \mathrm{C}$. Specific primers for each gene were designed and are listed in Table S3. The mRNA levels were quantified by normalizing their levels to the levels of the ACTIN 7 using the Ct method. All of the reactions were performed with three biological replicates. Each replicate was obtained by pooling tissue from 3 to 4 individual plants.

\section{Oil body cell and oil body distance measurement}

For live imaging, gemmalings were grown on standard medium in small Petri dishes ( $6 \mathrm{~cm}$ diameter). 3 day-old gemmalings were stained with BODIPY 493/503 working solution (dissolved in water) for $3 \mathrm{~min}$. Approximately $30 \mu \mathrm{L}$ dye was used to cover a gemmaling. Samples were rinsed with water two times, covered with water and observed using Leica TCS SP8 confocal microscope and $25 x$ 0.95 NA water dip-in objective. To observe fluorescence, $561 \mathrm{~nm}$ excitation and $570-600 \mathrm{~nm}$ emission was used for tdTomato and $488 \mathrm{~nm}$ excitation and $503-533 \mathrm{~nm}$ emission was used for BODIPY in sequential scan mode. All samples were imaged using the same settings. Images were analyzed in Fiji software. First, all stacks were trimmed to the same size relative to the estimated position of the apical cell along the $z$ axis. Prior to applying "sum slices" projection to the trimmed stacks, black and white pixels were added in a lower left corner of each slice as a calibration for lowest and highest possible values. In these projections, some nuclei visually stood out as much brighter than the surrounding nuclei. To visualize only these nuclei, all projections were processed as follows: format was set to 16 bit, intensities were multiplied by 100 and smoothed once. For tdTomato channel, brightness \& contrast values were set to show only the pixels with intensity value of 10000 or more. To assess the distances of bright nuclei and oil bodies from the apical notch, distance bins were created using Concentric circles plug-in. Circles were centered in the middle of the apical notch of each image and placed in $25 \mu \mathrm{m}$ distance intervals. Oil bodies and bright nuclei were quantified for each of the distance bins. Data was visualized and statistically analyzed in $\mathrm{R}$. To visualize apical notch surface in 3D, a surface mesh was created in MorphoGraphX program using signal from BODIPY channel [89].

\section{Oil body cell tracking}

For live imaging, gemmalings were grown on normal media in small Petri dishes (6 cm diameter). A few droplets of water were added around the rim of the medium 30 min before imaging, to soak the agar and prevent sample movements during the imaging. Gemmalings were stained with BODIPY $493 / 503$ working solution (dissolved in water) for 5 min. Approximately $30 \mu \mathrm{L}$ dye was used to cover a 5-day-old gemmaling. $15 \mu \mathrm{L}$ of $0.1 \%$ propidium iodide (PI) was added directly to BODIPY and removed after $30 \mathrm{~s}$. Samples were rinsed with water three times, covered with water and observed using Leica TCS SP8 confocal microscope and 25x 0.95 NA water dip-in objective. To observe fluorescence, $488 \mathrm{~nm}$ excitation and $503-523 \mathrm{~nm}$ emission was used for BODIPY, $552 \mathrm{~nm}$ excitation and $560-580 \mathrm{~nm}$ emission was used for tdTomato, and $552 \mathrm{~nm}$ excitation and $620-640 \mathrm{~nm}$ emission was used for PI. The two lasers were used in sequential scan (between stacks), and Lightning mode was used for acquisition. Standard deviation projections of $z$ stacks were generated in Fiji and used for oil body cell tracking. In case of doubt about the identity or lineage of an object, the stacks were observed in MorphoGraphX.

\section{Plant stress treatments}

Wild-type and Mpc1hdz mutant gemmae were grown in agar plates containing normal media. After 1-week growth in control conditions, each plant was transferred with tweezers to a new plate or pot to be exposed to different treatments. Plant areas were recorded before and after the treatment to estimate the impact on the relative growth rate of each genotype. Plant growth rate is the proportion between final and initial area of the thallus. Pictures of whole plants were taken at different time intervals using a Leica 
MZ10F stereomicroscope. Thallus area was quantified from pictures with ImageJ software. For cold treatments and high light intensity (HLI) plants were transferred to plates containing the normal media (3 plates with 12 plants for treatment and 3 plates as control). Control plates remained in control conditions. Cold treatment was performed at $4^{\circ} \mathrm{C}$ in a chamber with similar light and photoperiod conditions and observed after 1 week. HLI treatment was performed in the same chamber but $1000 \mu \mathrm{mol} \mathrm{m}^{-2} \mathrm{~s}^{-1} \mathrm{LED}_{\text {lights with the }}$ same photoperiod and observed after 1 week. For UV treatment plants were transferred to normal media and exposed to a laminar flow bench UV-C light for $1 \mathrm{~h}$ with the lid open (UV+) or closed (UV-), after that they were recovered in control conditions and observed after 1-week. For osmotic stress, normal media was supplemented with solutions of $\mathrm{NaCl}$ or mannitol at a $50 \mathrm{mM}$ final concentration or water (mock). Plants were grown in control conditions for 18 days. For starvation, plants were transferred to 1/100 Gamborg's B5 media (1\% (w/v) agar, pH 5.5), grown in control conditions, and observed after 2 weeks (normal media was used as control). For nonaxenic growth, plants were transplanted to pots with rockwool as substrate in a growth chamber in similar temperature and photoperiod as control conditions with irrigation.

The water loss experiment was performed as in Re et al. [90]. Briefly, 15-day-old wild-type and Mpc1hdz plants grown in control conditions and normal media were detached from the agar, placed in a paper towel, and weighed every $30 \mathrm{~min}$ during $2 \mathrm{~h}$ at room temperature. The percentage (\%) of water loss was calculated as follows: [initial weight - weight]/ [initial weight] $\times 100$.

\section{Pill bug feeding assay}

Feeding assay was performed according to Nakazaki et al. [91], with slight modification. Gemmae were cultured on 1/2 Gamborg's B5 medium (Nihon Pharmaceutical, Tokyo, Japan, Cat\#399-00621) containing 1\%(w/v) agar (Nacalai tesque, Kyoto, Japan, Cat\#01028-85) and 1\%(w/v) sucrose (Wako Pure Chemical, Osaka, Japan, Cat\#196-00015) for 5 days at $22^{\circ} \mathrm{C}$ under continuous white fluorescent light $\left(50 \mu \mathrm{mol} \mathrm{m} \mathrm{m}^{-2} \mathrm{~s}^{-1}\right)$. Five-day-old thalli were transferred onto $1 / 2 \times$ Gamborg's B5 medium containing $1 \%(\mathrm{w} /$ v) agar without sucrose and cultured for additional 5 days under the same condition above, or 8 days in case of BoGa ecotype. Pill bugs (Armadillidium vulgare) were collected at the Myodaiji area of National Institute for Basic Biology (Aichi, Japan). Before the assay, pill bugs were maintained on Prowipe (Daio Paper, Tokyo, Japan) moistened with sterilized water for $48 \mathrm{~h}$ at $22^{\circ} \mathrm{C}$ under dark condition to be starved. Six pill bugs were introduced into each medium plate containing the 10-day-old thalli, or 13-day-old thalli in case of BoGa ecotype, and kept together for $24 \mathrm{~h}$ under the dark condition at $22^{\circ} \mathrm{C}$. The thallus areas of $M$. polymorpha were calculated using ImageJ (National Institute of Health, https://imagej.nih.gov/ij/), and figures were processed with Photoshop software (Adobe systems, USA).

\section{Ethanol extraction and overlay assay}

Wild-type and Mpc1hdz gemmae were grown in agar plates containing normal media and control then transferred to pots using soil and vermiculite (1:1 parts) and grown in control conditions with irrigation for one month. Plant materials were snap-frozen with liquid $\mathrm{N}_{2}$, and ground using a mortar and pestle ( $\sim 5 \mathrm{~g}$ fresh weight). The plant powder was extracted with EtOH for $24 \mathrm{~h}$ in agitation. Debris was removed by filtration and removal of solvent was performed using a SpeedVac. The stock solution of crude extracts was prepared as $10 \mathrm{mg} \mathrm{mL}^{-1}$ in DMSO. Bacillus subtilis overlay assay was performed as described before [92]. Briefly, $3 \mathrm{~mL}$ of liquid LB media is inoculated with $30 \mu \mathrm{L}\left(1 / 100\right.$ dilution) of an OV culture of Bacillus subtilis and grown for $4 \mathrm{~h}$ at $28^{\circ} \mathrm{C}$ with agitation. Then, $3 \mathrm{~mL}$ of soft agar is inoculated using $100 \mu \mathrm{L}$ of the bacterial culture and plated as a uniform layer on a Petri dish containing solid LB media. Finally, $10 \mu \mathrm{L}$ of liquid plant extract is spotted on the top of the agar plate. The plates were incubated overnight at $28^{\circ} \mathrm{C}$ and the colony-growth inhibition was scored the next morning. For quantification purposes, pictures of bacterial growth inhibition were taken using stereoscopic microscope (Leica MZ10F) and calculated as density of colonies.

\section{RNA-seq}

Total RNA isolated from 3-week-old thalli (QIAGEN RNAeasy Plant Kit) from each genotype was assessed for quality with a Nanodrop spectrophotometer and a Bioanalyzer 2100 microfluidics system (Agilent 6). For expression analysis in control conditions, one sample per genotype was used (Mpc1hdz-1 ${ }^{g e}$, Mpc1hdz-2 ${ }^{g e}$, WT female, WT male) and mutant and wild-type were treated as replicates. For expression in non-axenic conditions, plants were grown as described before and three biological replicates per genotype were taken from Mpc1hdz-2 ${ }^{g e}$ and WT female. Library preparation substrates were enriched for mRNA (Illumina TruSeq Stranded mRNA technology). Sequencing used the Illumina NextSeq500 7 in HighOutput mode (20 million single-ended 75 b reads per sample). Reads were mapped onto the Marchantia v3.1 (http://marchantia.info/) assembly using TopHat v2.1.0 for Galaxy [78]. Resulting BAM files were loaded onto IGV 1.3.1 to produce Sashimi Plots [79]. A count matrix of average raw reads/gene/sample was created using HTseq-code using Galaxy [80]. Raw reads were normalized by total million reads per library (RPM) and transcript lengths in kb (RPKM). The edgeR package was used for differential gene expression analysis using Galaxy [81]. Summary statistics of RNA-seq analysis are available in Table S4.

For GO term and protein family enrichment analysis, the algorithm and annotations were as described previously [93]. Gene names from Marchantia v5.1 [94] was assigned in accordance to gene corresponding table available at http://marchantia.info/ nomenclature/ [2]. Genes were annotated using information nomenclature previously published [95] or available at MarpolBase.

Hexane extraction and gas chromatography

Samples of WT and transgenic plants (3-4 samples per genotype) were collected from 3-week-old plants in different treatments and snap frozen in liquid nitrogen. Each sample was ground with a pestle in a mortar containing liquid nitrogen. $300 \mu \mathrm{L}$ of $\mathrm{n}$-hexane was 
added to $\sim 400 \mathrm{mg}$ of ground tissue, centrifuged and extracted overnight with shaking at $100 \mathrm{rpm}$. For samples in starvation, extraction was scaled-down to $50 \mu \mathrm{L}$ of solvent and $\sim 50 \mathrm{mg}$ of grounded tissue. Each extract was then vortexed and centrifuged, and the supernatant was transferred into glass vials for gas chromatographic analyses.

Constituents in hexane extracts were identified by GC-MS and quantified by GC-FID. The GC-MS system comprised a Gerstel 2.5.2 autosampler, a 7890A gas chromatograph and a 5975C quadrupole MS (Agilent Technologies, Santa Clara, USA). Aliquots of extracts $(1 \mu \mathrm{L})$ were injected in splitless mode and the MS was adjusted to manufacturer's recommendations using tris-(perfluorobutyl)-amine (FC-43). We used the following MS source conditions: injection temperature $250^{\circ} \mathrm{C}$, transfer line $280^{\circ} \mathrm{C}$, ion source $230^{\circ} \mathrm{C}$, quadrupole $150^{\circ} \mathrm{C}, 70 \mathrm{eV}$ (El mode), 2.66 scans s $^{-1}$ and scanning range $\mathrm{m} / \mathrm{z}$ 50-600. The GC-MS column was a VF-5MS (30 $\mathrm{m} \times 250 \mu \mathrm{m}$ i.d. with $0.25 \mu \mathrm{m}$ film thickness) fitted with a $10 \mathrm{~m}$ EZ-Guard column (J \& W, Agilent). Helium was used as the carrier gas at a flow rate of $1 \mathrm{~mL} \mathrm{~min}^{-1}$. The column temperature was held at $70^{\circ} \mathrm{C}$ for 6 min following injection, then ramped at $10^{\circ} \mathrm{C}$ min ${ }^{-1}$ to $320^{\circ} \mathrm{C}$ and held at that temperature for $5 \mathrm{~min}$. Mass spectra were evaluated using Agilent MSD ChemStation (E.02.02.1431) and sesquiterpenes were identified using a mass spectra library described before [82]. Other constituents were identified using the NIST 11 database (https://chemdata.nist.gov/dokuwiki/doku.php?id=chemdata:start). Constituent identity was confirmed by their relative retention times and by comparison to authentic standards where available, including the FAME mix (Sigma-Aldrich, St. Louis, USA).

Quantification of constituents in extracts was performed using a GC-FID system comprising a Perkin Elmer Autosystem XC (Perkin Elmer, Melbourne, Australia) fitted with a low polarity Zebron ZB-5 (30 m $\times 250 \mu \mathrm{m}$ i.d., Phenomenex) column. Aliquots of extracts $(2 \mu \mathrm{L})$ were injected under a split flow of $7.5 \mathrm{~mL} \mathrm{He} \mathrm{min}^{-1}$. The injector and detector temperatures were 275 and $320^{\circ} \mathrm{C}$, respectively. The temperature program was the same as for the GC-MS analyses. Quantification of constituents in each class was based on calibration series of commercial standards of $d$-limonene (monoterpenes and $\mathrm{C}_{8}$ volatiles), aromadendrene (aromadendrane sesquiterpene hydrocarbons), valencene (other bicyclic sesquiterpene hydrocarbons), caryophyllene oxide (oxygenated sesquiterpenes), hexadecane (hydrocarbons), palmitic acid (fatty acids and aldehydes), phytol (diterpenes) and stigmasterol (phytosterols; Sigma-Aldrich). A summary of GC-FID analysis of plant extracts is shown in Data S1.

\section{QUANTIFICATION AND STATISTICAL ANALYSIS}

Statistical significance was calculated using ANOVA from agricolae package using Rstudio software (https://www.rstudio.com) and corrected by Tukey HSD (alpha $=0.05$ ) for levels calculations. In other cases, built-in packages in R were used for pairwise $t$ test, Wilcoxon rank sum test and binominal test. In each figure it is explained which test was used. 